\title{
PATHWAYS FOR EMOTION: INTERACTIONS OF PREFRONTAL AND ANTERIOR TEMPORAL PATHWAYS IN THE AMYGDALA OF THE RHESUS MONKEY
}

\author{
H. T. GHASHGHAEI ${ }^{\mathrm{a}}$ and H. BARBAS ${ }^{\mathrm{a}, \mathrm{b} *}$ \\ ${ }^{a}$ Department of Health Sciences, Boston University, 635 Commonwealth Avenue, Room 431, Boston, MA 02215, USA \\ ${ }^{b}$ New England Regional Primate Research Center, Harvard Medical School, Southborough, MA, USA
}

\begin{abstract}
The amygdala has been implicated in processing information about the emotional significance of the environment and in the expression of emotions, through robust pathways with prefrontal, anterior temporal areas, and central autonomic structures. We investigated the anatomic organization and intersection of these pathways in the amygdala in rhesus monkeys with the aid of bidirectional, retrograde and anterograde tracers. Connections of the amygdala with orbitofrontal and medial prefrontal areas were robust and bidirectional, whereas connections with lateral prefrontal areas were sparse, unidirectional and ascending. Orbitofrontal axons terminated densely in a narrow band around the borders of the magnocellular basolateral nucleus, surrounded by projection neurons along a continuum through the nuclei of the basal complex. In contrast, the input and output zones of medial prefrontal areas were intermingled in the amygdala. Moreover, medial prefrontal axonal terminations were expansive, spreading into the parvicellular basolateral nucleus, which is robustly connected with hypothalamic autonomic structures, suggesting that they may influence the expressive emotional system of the amygdala. On the other hand, orbitofrontal axons heavily targeted the intercalated masses, which issue inhibitory projections to the central nucleus, at least in rats and cats. The central nucleus, in turn, issues a significant inhibitory projection to hypothalamic and brainstem autonomic structures. This evidence suggests that orbitofrontal areas exercise control on the internal processing of the amygdala. In addition, the results provided direct evidence that the connections of anterior temporal visual and auditory association cortices occupy overlapping territories with the orbitofrontal cortices particularly in the posterior half of the amygdala, and specifically within the intermediate sector of the basolateral nucleus and in the magnocellular part of the basomedial nucleus (also known as accessory basal), suggesting a closely linked triadic network. This intricate network may be recruited in cognitive tasks that are inextricably linked with emotional associations.
\end{abstract}

C 2002 IBRO. Published by Elsevier Science Ltd. All rights reserved.

Key words: orbitofrontal, medial prefrontal, cognition-emotion, primate, hypothalamic autonomic centers.

The amygdala has a role in emotions through participation in two key functions: Processing signals about the emotional significance of the environment, and interfacing with autonomic motor systems for the expression of emotions (for reviews see Damasio, 1994; Gallagher and Holland, 1994; Alheid and Heimer, 1996; Davis, 1997; LeDoux, 2000). These functions are processed along robust pathways linking the amygdala with orbitofrontal, medial prefrontal, anterior temporal cortices, and central autonomic structures. The amygdala and the orbitofrontal cortex, in particular, have common features (e.g. Schoenbaum et al., 2000; for reviews see Barbas, 2000; Bechara et al., 2000). For example, both structures receive massive projections from cortices representing each of the sensory modalities, originating from highorder association areas specialized in processing features of stimuli and their memory (for reviews see De Olmos, 1990; Barbas, 1995a; McDonald, 1998; Cavada et al.,

*Correspondence to: H. Barbas, Department of Health Sciences, Boston University, 635 Commonwealth Avenue, Room 431, Boston, MA 02215 , USA. Tel.: +1-617-353-5036; fax: +1-617-353-7567.

E-mail address: barbas@bu.edu (H. Barbas).

Abbreviations: AAA, anterior amygdaloid nuclei; AChE, acetyl cholinesterase; ACo nucleus, anterior cortical nucleus; AHA, amygdalohippocampal area; BDA, biotinylated dextran amine; BL, basolateral; BLmc, magnocellular part of BL nucleus; BLpc, parvicellular part of BL nucleus; BM, basomedial (or accessory basal); BMmc, magnocellular part of BM nucleus; $\mathrm{BMpc}$, parvicellular part of BM nucleus; $\mathrm{CB}$, calcium binding protein calbindin; $\mathrm{Cd}$, caudate nucleus; Ce nucleus, central nucleus; Co nucleus, cortical nucleus; En, endopiriform nucleus; EN, entorhinal cortex; FB, fast blue; FE, fluoroemerald; FR, fluororuby; HRP-WGA, horseradish peroxidase to wheat germ agglutinin; BLi, intermediate division of the BL nucleus; IM, intercalated masses; L nucleus, lateral nucleus; LA, lateral hypothalamic area; Me nucleus, medial nucleus; nBM, nucleus basalis of Meynert; O12, orbital area 12; O14, orbital area 14; OPAll, orbital periallocortex (agranular cortex); OPro, orbital proisocortex (dysgranular cortex); OT, optic tract; PA, posterior hypothalamic nucleus; PBS, phosphate-buffered saline; PCo, posterior cortical nucleus; Pir, piriform cortex; Put, putamen; PV, calcium binding protein parvalbumin; STG, superior temporal gyrus; TE, anterior temporal visual association area; TS1, anterior temporal auditory association area; TS2, temporal auditory association area; V46, ventral area 46; VCo nucleus, ventral cortical nucleus. 
2000). The amygdala and the orbitofrontal cortex thus appear to have a global overview of the environment, which likely is necessary for processing and remembering the emotional significance of stimuli.

The amygdala, orbitofrontal cortex, and medial prefrontal cortices also have key roles in the expression of emotions by innervating hypothalamic and brainstem autonomic structures (for reviews Price et al., 1987; De Olmos, 1990). Disruption of these pathways seriously affects emotional expression, as exemplified by the inability of patients with orbitofrontal lesions to respond autonomically in emotional situations (Damasio et al., 1990; for review see Kling and Steklis, 1976). Even though such patients have intact cognitive function, they make poor decisions, suggesting functional disconnection of cognitive processes from emotionally driven autonomic responses (Bechara et al., 1996).

The role of the amygdala in emotions thus appears to depend on an intricate network involving prefrontal, temporal and central autonomic structures. The organization and interaction of some key components of this network, however, are not known. For example, in contrast to the wealth of information on the projections from the amygdala to prefrontal cortices (e.g. Nauta, 1961; Jacobson and Trojanowski, 1975; Porrino et al., 1981; Barbas and De Olmos, 1990; Morecraft et al., 1992; Carmichael and Price, 1995a), little is known about pathways in the reverse direction in primates (Pandya et al., 1973; Aggleton et al., 1980; Van Hoesen, 1981; Carmichael and Price, 1995a; Chiba et al., 2001), which likely influence the activity of the amygdala in behavior. Here we addressed the extent to which components of these pathways intersect in the amygdala by investigating: (1) the organization of descending projections from prefrontal cortices, and their relationship to output from the amygdala to prefrontal cortices; (2) whether prefrontal and anterior temporal cortices have common sites of connections in the amygdala, that may form the structural basis to evaluate the emotional significance of the environment; (3) whether orbitofrontal and medial prefrontal cortices share zones of influence with hypothalamic autonomic structures in the amygdala. We provide evidence that specific orbitofrontal, medial prefrontal and temporal cortical pathways project onto excitatory and inhibitory pathways in the amygdala, and likely interact in fundamental mechanisms in the perception, memory and expression of emotions.

\section{EXPERIMENTAL PROCEDURES}

\section{Surgical procedures}

Experiments were conducted on 26 rhesus monkeys (Macaca mulatta), obtained through the Washington and New England Regional Primate Research Centers. Experiments were conducted according to the NIH guide for the Care and Use of Laboratory Animals (NIH publication 86-23, revised 1987), and all efforts were made to minimize animal suffering and to reduce their number. The monkeys were anesthetized with ketamine hydrochloride (10-15 mg/kg, intramuscularly) followed by sodium pentobarbital administered intravenously through a femoral catheter, or gas anesthetic (isofluorane), until a surgical level of anesthesia was accomplished. Surgery was performed under aseptic conditions while heart rate, muscle tone, respiration, and pupillary dilatation were closely monitored. For cortical injections, a craniotomy was made while the head was stabilized in a head holder, the dura cut and the cortex exposed.

The coordinates for injections in the hypothalamus were calculated using MR imaging. The animal was placed in a nonmetallic stereotaxic apparatus and imaged under a 1.5 Tesla MRI machine. The hollow tips of the ear bars of the stereotaxic apparatus were filled with $0.5 \%$ cupric sulfide or betadine salve, and were used as landmarks to calculate the coordinates for the depth of penetration to the hypothalamus. At the time of surgery the monkey was placed in the same stereotaxic apparatus and a small region of the cortex above the desired target was exposed. Surgery was then conducted to inject neural tracers, as described above. Injections were made in the right or left hemisphere, as indicated in Table 1 .

Injection of neural tracers. To study the zones in the amygdala connected with prefrontal cortices, we injected in prefrontal cortices bidirectional tracers (HRP-WGA, horseradish peroxidase conjugated to wheat germ agglutinin, Sigma, cat. \# 61769 , St. Louis, MO, USA; BDA, biotinylated dextran amine, Molecular Probes, cat. \# D-7135, Eugene, OR, USA) or anterograde tracers $\left(\left[{ }^{3} \mathrm{H}\right]\right.$ amino acids, New England Nuclear, Boston, MA, USA; Table 1). In each of 15 animals, HRP-WGA or BDA was injected in one prefrontal area using a microsyringe (Hamilton, $5 \mu \mathrm{l}$ ) mounted on a microdrive. BDA and HRPWGA are bidirectional tracers, which allowed us to establish input/output zones in the amygdala connected with different

Table 1. Summary of cases, injection sites, and tracers used

\begin{tabular}{|c|c|c|}
\hline Case name & Injection site(s) & Dye(s) \\
\hline \multicolumn{3}{|c|}{ Orbitofrontal } \\
\hline $\mathrm{MAR}^{\mathrm{a}} 1$ & OPro & {$\left[{ }^{3} \mathrm{H}\right] \mathrm{amino}$ acids } \\
\hline $\mathrm{AF}^{\mathrm{a}, \mathrm{b}} 1$ & OPro & HRP \\
\hline $\mathrm{AG}^{\mathrm{a}, \mathrm{b}} 1$ & OPAll/OPro & HRP \\
\hline $\mathrm{MBY}^{\mathrm{a}, \mathrm{b}} \mathrm{r}$ & $\mathrm{O} 12$ & HRP \\
\hline $\mathrm{MBC}^{\mathrm{a}} \mathrm{r}$ & $\mathrm{O} 14,11,13$ & {$\left[{ }^{3} \mathrm{H}\right]$ amino acids } \\
\hline $\mathrm{MBJ}^{\mathrm{a}, \mathrm{b}} \mathrm{r}$ & 11 & HRP \\
\hline $\mathrm{MFT}^{\mathrm{a}} 1$ & 11 & {$\left[{ }^{3} \mathrm{H}\right]$ amino acids } \\
\hline $\mathrm{AM}^{\mathrm{a}, \mathrm{b}} 1$ & 11 & HRP \\
\hline \multicolumn{3}{|c|}{ Medial prefrontal } \\
\hline $\mathrm{AH}^{\mathrm{a}, \mathrm{b}} \mathrm{r}$ & 25 & HRP \\
\hline $\mathrm{AE}^{\mathrm{a}, \mathrm{b}} 1$ & 32 & HRP \\
\hline $\mathrm{AY} \mathrm{Y}^{\mathrm{a}, \mathrm{b}} 1$ & 32 & BDA \\
\hline $\mathrm{MDQ}^{\mathrm{a}} 1$ & 32 & {$\left[{ }^{3} \mathrm{H}\right]$ amino acids } \\
\hline $\mathrm{AO}^{\mathrm{a}, \mathrm{b}} 1$ & M9 & HRP \\
\hline \multicolumn{3}{|c|}{ Lateral prefrontal } \\
\hline $\mathrm{AC}^{\mathrm{a}, \mathrm{b}} \mathrm{r}$ & D8 & HRP \\
\hline $\mathrm{AD}^{\mathrm{a}, \mathrm{b}} \mathrm{r}$ & D8 & HRP \\
\hline $\mathrm{AB}^{\mathrm{a}, \mathrm{b}} \mathrm{r}$ & D46 & HRP \\
\hline $\mathrm{MBH}^{\mathrm{a}, \mathrm{b}} \mathrm{r}$ & V46 & HRP \\
\hline $\mathrm{MFF}^{\mathrm{a}}$ & V46 & {$\left[{ }^{3} \mathrm{H}\right]$ amino acids } \\
\hline $\mathrm{AA}^{\mathrm{a}, \mathrm{b}} \mathrm{r}$ & V46 & HRP \\
\hline $\mathrm{SF}^{\mathrm{a}, \mathrm{b}} \mathrm{r}$ & D10 & HRP \\
\hline \multicolumn{3}{|c|}{ Temporal cortices } \\
\hline $\mathrm{AV}^{\mathrm{a}, \mathrm{b}} 1$ & TE1/TS1 & BDA \\
\hline $\mathrm{AT}^{\mathrm{a}, \mathrm{b}} \mathrm{r}$ & 36 & $\mathrm{BDA}$ \\
\hline \multicolumn{3}{|c|}{ Temporal and orbitofrontal cortices } \\
\hline $\mathrm{BA}^{\mathrm{a}, \mathrm{b}} 1$ & TE, O12 & FR, FB \\
\hline $\mathrm{BC}^{\mathrm{b}} \mathrm{r}$ & TS2, OPro, 13 & $\mathrm{FR}, \mathrm{FB}, \mathrm{FE}$ \\
\hline \multicolumn{3}{|c|}{ Hypothalamus } \\
\hline $\mathrm{AW}^{\mathrm{b}} \mathrm{r}$ & LA & FB \\
\hline $\mathrm{AX}^{\mathrm{a}, \mathrm{b}} 1$ & PA & BDA \\
\hline
\end{tabular}

anterograde analysis.

${ }^{\mathrm{b}}$ Retrograde analysis.

1, left hemisphere; $r$, right hemisphere; D8, dorsal area 8; D10, dorsal area 10; D46, dorsal area 46; M9, medial area 9; V46, ventral area 46. 
cortices. BDA labels the entire extent of axonal terminals and boutons. $\left[{ }^{3} \mathrm{H}\right]$ amino acids, which are anterograde tracers, were injected in the prefrontal cortices of five animals to investigate prefrontal axonal terminations in the amygdala.

We compared temporal cortical zones in the amygdala with those connected with prefrontal cortices with the aid of retrograde and anterograde tracers injected in the respective cortices (fluororuby (FR), dextran tetramethylrhodamine, MW 3000, Molecular Probes, cat. \# D3308; BDA; fast blue (FB), Sigma, cat. \# F5756; fluoroemerald (FE), dextran fluorescein, MW 10000, cat. \# D1820, Molecular Probes). Injections in temporal areas were restricted to anterior sectors, known to be heavily linked with orbitofrontal and medial prefrontal cortices, as well as with the amygdala (e.g. Barbas, 1993; Carmichael and Price, 1995b; Stefanacci et al., 1996; Barbas et al., 1999; RempelClower and Barbas, 2000). This comparison was first made across cases by injecting BDA in anterior temporal visual association area /anterior temporal auditory association area (TE1/ TS1) of one monkey and in area 36 of another monkey (cases $\mathrm{AV}$ and AT, Table 1). We then made direct comparisons in two cases after injections of distinct fluorescent tracers in both orbitofrontal and temporal areas (cases BA and BC). This method allowed simultaneous visualization of the populations of neurons in the amygdala projecting to temporal and orbitofrontal cortices. In one of these cases (case BA) it was also possible to visualize anterograde label from temporal area TE in the amygdala, with the aid of the tracer FR.

Finally, we included a few observations on the comparison of the cortical zones of the amygdala with zones that project to some hypothalamic autonomic centers. The latter were studied after injecting the fluorescent dye FB in the lateral hypothalamic area (LA, case AW) and BDA in the posterior hypothalamic nucleus (PA, case AX; Table 1), which are associated with autonomic functions (for reviews see Saper, 1990; Loewy, 1991).

The needle was lowered to a selected cortical site under microscopic inspection. For cortical injections, small amounts of the injectate $(0.05-0.1 \mu \mathrm{l}, 8 \%$ HRP-WGA; $0.4 \mu \mathrm{l}, 3 \%$ FB; $0.8 \mu \mathrm{l}$, $10 \%$ FR and FE; $0.4-2.0 \mu \mathrm{l},\left[{ }^{3} \mathrm{H}\right]$ leucine and $\left[{ }^{3} \mathrm{H}\right]$ proline, specific activity 40-80 $\mu \mathrm{Ci} ; 1-2 \mu \mathrm{l}$ BDA) were delivered $1.5 \mathrm{~mm}$ below the pial surface at each of two adjacent sites separated by 1-2 $\mathrm{mm}$. In the case of injections in the hypothalamus, one to two penetrations were made from the top of the brain to the calculated depths. A period of 10-15 min was allowed for each injection for adequate dye penetration at the injection site to minimize spreading of the dyes during retraction of the needle.

\section{Perfusion and tissue processing}

Tritiated amino acid experiments. The animals injected with $\left[{ }^{3} \mathrm{H}\right]$ amino acids were given an overdose of anesthetic (sodium pentobarbital, $>50 \mathrm{mg} / \mathrm{kg}$, to effect) and perfused with $10 \%$ formalin after 10 days of survival. The brain was removed from the skull, photographed, embedded in paraffin, cut in $10 \mu \mathrm{m}$ coronal sections, and mounted on glass slides. The processing of the sections was according to the autoradiographic method (Cowan et al., 1972). Sections were counterstained with Thionin (Fisher, cat. \# T-409) and coverslipped.

HRP, fluorescent dyes, and BDA injections. Following a survival period of $40-48 \mathrm{~h}$ animals injected with HRP-WGA were given a lethal dose of anesthetic (sodium pentobarbital, > $>0$ $\mathrm{mg} / \mathrm{kg}$, to effect) and perfused through the heart with saline followed by 21 of fixative $(1.25 \%$ glutaraldehyde, Fisher, cat. \# 02957-1, 1\% paraformaldehyde, Fisher, cat \# 04042-500 in $0.1 \mathrm{M}$ phosphate buffer, $\mathrm{pH}$ 7.4). The fixative was followed by perfusion with 21 of cold $\left(4^{\circ} \mathrm{C}\right)$ phosphate buffer $(0.1 \mathrm{M}, \mathrm{pH}$ 7.4). The brain was then removed from the skull, photographed, and placed in glycerol phosphate buffer [10\% glycerol, Sigma, cat. \# G7757; 2\% dimethyl sulfoxide (DMSO), Sigma, cat. \# D5879, in 0.1 M phosphate buffer, pH 7.4] for one day and then in $20 \%$ glycerol phosphate buffer for two additional days.

The survival period for animals with fluorescent dyes and BDA injections was 14-18 days. The animals were then anesthetized and perfused with $4 \%$ paraformaldehyde in $0.1 \mathrm{M}$ phos- phate-buffered saline (PBS) (pH 7.4). The brain was removed from the skull, photographed and placed in graded solutions of sucrose (10-30\%, Fisher, S5-500 in 0.1 M PBS) for cryoprotection.

Brains were frozen in $-75^{\circ} \mathrm{C}$ isopentane (Fisher, cat. \# 035514) and cut on a freezing microtome in the coronal plane at 40 $\mu \mathrm{m}$ in 10 series. In the HRP-WGA and BDA experiments, one series was treated to visualize HRP (Mesulam et al., 1980) or BDA (described below). The tissue was mounted, dried, and counterstained with Neutral Red (Fisher, cat. \# N129, for HRP) or Thionin (for BDA). Adjacent series of sections were stained for Nissl bodies, myelin, or acetyl cholinesterase (AChE) to aid in delineating architectonic borders (Geneser-Jensen and Blackstad, 1971; Gallyas, 1979).

In experiments with fluorescent dye injections, two series of sections were mounted for mapping labeled neurons. One series was coverslipped with Fluoromount (Fisher, cat. \# 23750008) and stored in light-tight boxes with Drierite at $4^{\circ} \mathrm{C}$. The second series was plotted to visualize and localize retrogradely labeled neurons under a microscope equipped with an epifluorescence attachment. After plotting, sections were counterstained with Thionin. In all cases adjacent series of sections were stained for myelin, AChE, or Nissl to aid in delineating architectonic borders in the cortex and in the amygdala.

Immunocytochemical procedures. In experiments with BDA injections, one series of sections was washed in $0.1 \mathrm{M}$ PBS and placed in avidin-biotin complex solution (Vector Labs, cat. \# PK 6100, Burlingame, CA, USA) overnight. The sections were then washed and processed either for immunoperoxidase reaction [e.g. 3,3'-diaminobenzidine (DAB), Zymed lab, cat. \# 00-2020] or for immunofluorescence using avidin conjugated probes for visualizing the transported dextran.

In order to simultaneously visualize neurons labeled with the tracer FB and neurons positive for calcium binding proteins we used standard immunocytochemical techniques to visualize calbindin $(\mathrm{CB}$, mouse monoclonal, Accurate Chem. and Scientific Corp., cat \# YSW3002) or parvalbumin (PV, mouse monoclonal, Chemicon, cat. \# MAB 1572). Sections through the amygdala, containing neurons labeled with FB, were washed in $0.1 \mathrm{M}$ PBS (three times, 10 min each) and placed in a blocking solution containing $10 \%$ goat serum (Vector Labs, cat. \# S1000, in $0.1 \mathrm{M}$ PBS and $0.3 \%$ Triton X-100, Sigma, cat. \# X-100). After $1 \mathrm{~h}$ incubation the sections were quickly rinsed in $0.1 \mathrm{M}$ PBS and placed in the primary antibody for CB or PV $(1: 2000)$, in $0.1 \mathrm{M}$ PBS containing $1 \%$ goat serum, for $48-72 \mathrm{~h}$ at $4^{\circ} \mathrm{C}$. The sections were then washed in $0.1 \mathrm{M}$ PBS (three times, $10 \mathrm{~min}$ each) and placed in the secondary antibody (cyanoindocarbocyanine, Cy3 conjugated goat anti-mouse IgG, Chemicon, cat. \# AP124C, 1:500 in 0.1 M PBS and 1\% goat serum). After overnight incubation, the sections were washed again in $0.1 \mathrm{M}$ PBS (three times, $10 \mathrm{~min}$ each). Some of these sections were counterstained with a fluorescent Nissl stain (NeuroTrace 530/615, Molecular Probes, cat. \# N21482) for 1-3 h, washed, and all sections were mounted on glass slides.

\section{Data analysis}

Mapping projection neurons. Sections ipsilateral to the injection sites were viewed under brightfield or fluorescence illumination to map retrogradely labeled neurons in the amygdala. The terminology for the amygdala was according to (Price et al., 1987; De Olmos, 1990) and nuclei were delineated using Nissl and AChE stains. Mapping of retrogradely labeled neurons was conducted using a microscope-computer interface and digital plotter using software developed in our laboratory, as described previously (e.g. Barbas et al., 1999). We also used a computerized system and motorized stage for mapping retrogradely labeled neurons (Neurolucida, MicroBrightField, Colchester, VT, USA).

Mapping anterograde label. We used phase-contrast and darkfield illumination to outline and map anterograde label in the amygdala in cases with injections of $\left[{ }^{3} \mathrm{H}\right]$ amino acids, HRP- 
WGA, and BDA. We used two methods to evaluate anterograde label. First, we rated anterograde label qualitatively by visual inspection as light $(+)$, moderate $(++)$, or dense $(+++)$. Second, we measured the regional density of anterograde label in the amygdala using an image analysis system (MetaMorph, Universal Imaging, West Chester, PA, USA), as described previously (e.g. Barbas and Rempel-Clower, 1997; Rempel-Clower and

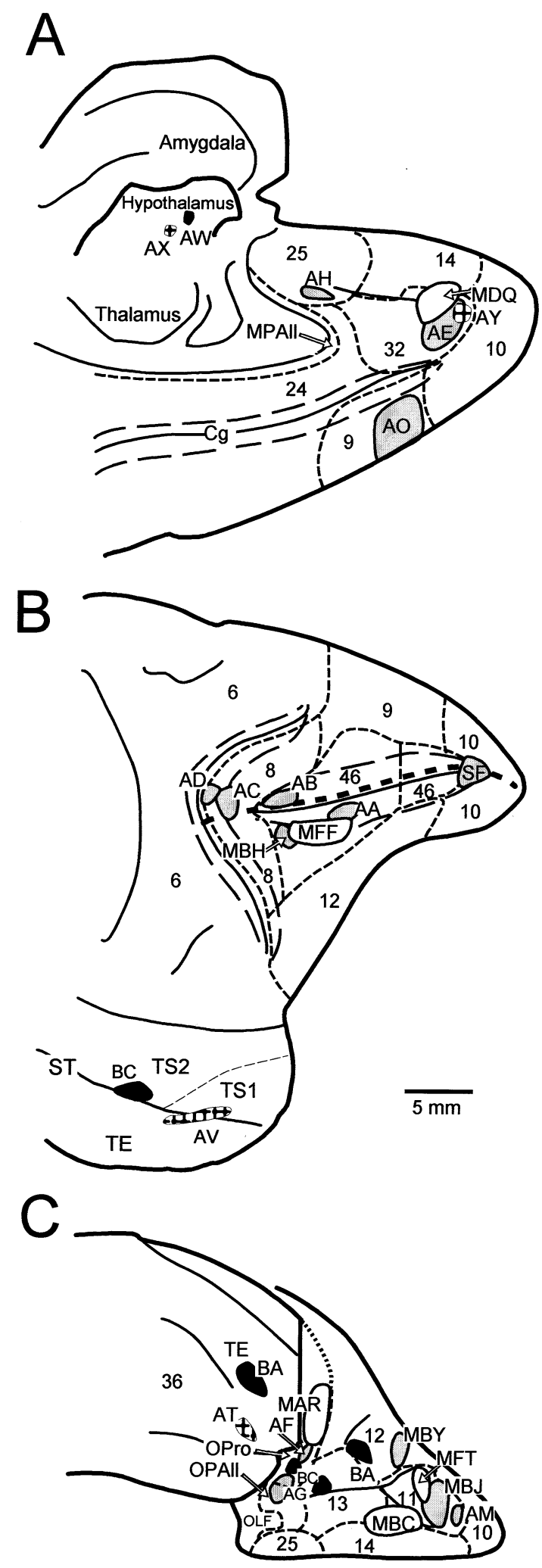

Barbas, 1998, 2000; Barbas et al., 1999; Ghashghaei and Barbas, 2001). The system uses a CCD camera mounted on the microscope to capture images directly from brain sections. Measurements of the density of anterograde label were made at $100 \times$ magnification under darkfield illumination, using a fiber optic illuminator which ensures even lighting at low and high magnification (Optical Analysis Corp., Nashua, NH, USA). Measurements of anterograde label were made systematically at eight rostrocaudal levels throughout the amygdala. To determine labeling above background level, measurements were taken from areas with no anterograde label in the same sections, and the values were subtracted from the density scores. Density measures were taken from five to six sites within an area, avoiding artifact and retrogradely labeled neurons. After subtraction of background, the range of intensity of label was divided into thirds, where the first division was considered light, the middle division moderate, and the upper division dense. These rating scores were converted to a scale of 0 to +++ , corresponding to 0 , no label; +, light label; ++, moderate label; and +++ , dense label. The density scores from the two methods were highly correlated (Pearson $r=0.98, P<0.001$ ). The density scores were then plotted in a three-dimensional surface plot showing the distribution of the scores in eight rostral to caudal levels of the amygdala in each case using Matlab software (MathWorks, Natick, MA, USA).

Photography. Photomicrographs for presentation of data were captured directly from histological brain slides using a CCD camera and the Neurolucida Virtual Slice software (MicroBrightField), and were imported into Adobe Photoshop (Adobe Systems Inc., San Jose, CA, USA) for assembly, labeling, and adjustment of overall brightness, but were not retouched. For presentation of doubly labeled sections, images captured under different fluorescent filters were imported into the same file in Photoshop, superimposed, and the opacity adjusted so that both images were visible.

\section{RESULTS}

\section{Injection sites and neural tracers}

Results were obtained from experiments with injections of neural tracers in several prefrontal cortices, and in a sample of anterior temporal areas, and autonomic hypothalamic structures, both linked with the amygdala as well as with prefrontal cortices. A composite diagram of the location of tracer injections is shown in Fig. 1, and the cases, types of dyes used, injection sites and hemisphere, and analyses conducted are presented in Table 1. Details for most injection sites in prefrontal architectonic areas, including their depiction in coronal sections, photographs, and serial reconstruction on twodimensional maps, were described previously in mapping of other structures (Barbas et al., 1991, 1999; Barbas,

Fig. 1. Composite of injection sites shown on the (A) medial; (B) lateral; and (C) orbital surfaces of the frontal and temporal lobes. The injection sites are superimposed on an architectonic map of the prefrontal and temporal cortices (Galaburda and Pandya, 1983; Barbas and Pandya, 1989). Dotted lines demarcate architectonic areas indicated by numbers. MPAll (medial periallocortex), OPAll (orbital periallocortex), OPro, OLF (olfactory area), TE, TS1, TS2, refer to architectonic areas; ST, superior temporal sulcus; $\mathrm{Cg}$, cingulate sulcus. All other letter combinations refer to cases. Patterns designating injection sites refer to the type of tracer used: black, fluorescent dyes; stripes, BDA; black outline, $\left[{ }^{3} \mathrm{H}\right]$ amino acids; gray, HRP-WGA. 
1993, 1995b; Dermon and Barbas, 1994; Barbas and Blatt, 1995; Rempel-Clower and Barbas, 1998), including a study restricted to description of projection neurons directed from the amygdala to the prefrontal cortex (Barbas and De Olmos, 1990). Injections of tracers included all layers of the injected cortices.

The anterograde tracers used (tritiated amino acids, an anterograde tracer; WGA-HRP and BDA, bidirectional tracers) differ in their resolution to differentiate terminal label from fibers of passage, and in the case of BDA in distinguishing anterograde label from axons of retrogradely labeled neurons. Nevertheless, the pattern of labeling we describe in this section was consistent regardless of the type of tracer used.

\section{Efferent projections from prefrontal cortices to the amygdala}

The density of axonal terminations from prefrontal, temporal and hypothalamic areas is shown for all cases in Table 2. We first studied the efferent or bidirectional connections with the amygdala of several prefrontal cortices, encompassing orbitofrontal $(n=8)$, medial $(n=5)$, and lateral prefrontal cortices $(n=7)$ (Table 1$)$.

Orbitofrontal termination zones. Axons from caudal orbitofrontal areas issued a dense projection to the amygdala. Evidence was obtained from three caudal orbitofrontal sites, whose labeled axons terminated heavily in and around the basolateral (BL) and basomedial (BM, also known as accessory basal) nuclei in a similar manner, both in topography and density (cases MAR; AF; AG; Figs. 2 and 3; Table 2). Labeled axonal terminals were strongest in the caudal half of the amygdala (Fig. 3, inset) and assumed a U-shaped pattern, in and around the borders of the magnocellular part of $\mathrm{BL}$ nucleus (BLmc), in the lateral (L), and BM nuclei, and the transition zone between the BLmc and parvicellular sectors of BL nucleus (BLpc) (Figs. 2 and 3). Labeled axonal terminals of light to moderate density were noted in the magnocellular part of BM nucleus (BMmc) (Figs. 2F, 3, cases AF, AG; Table 2). Heavy to moderate axonal terminals were also noted in the anterior amygdaloid area (AAA) and the intercalated masses (IM), most notably between the BL and the central (Ce) nuclei caudally (Fig. 2F, case AF, C; Fig. 3, case AG, C-D), and in the Ce nucleus in two of the cases (Fig. $2 F$, cases MAR and AF, Fig. 3, case AF, C).

Anterior orbitofrontal areas $\mathrm{O} 14, \mathrm{O} 12$ and 11, on the other hand, issued a light projection in comparison with caudal orbitofrontal areas (Fig. 3, inset; Fig. 4; Table 2.). Light anterograde label from axons originating in

Table 2. Distribution and density of axonal terminals from prefrontal, temporal, and hypothalamic areas in the amygdala

\begin{tabular}{|c|c|c|c|c|c|c|c|c|c|c|c|c|c|c|c|c|c|}
\hline \multirow{2}{*}{$\begin{array}{l}\text { Case } \\
\text { Orbitofrontal }\end{array}$} & \multirow[t]{2}{*}{ Injection sites } & \multicolumn{16}{|c|}{ Amygdaloid nuclei } \\
\hline & & \multicolumn{2}{|l|}{$\mathrm{L}$} & \multicolumn{3}{|l|}{ BL } & \multicolumn{2}{|l|}{$\mathrm{BM}$} & \multicolumn{3}{|c|}{$\mathrm{Co}$} & $\mathrm{Me}$ & \multicolumn{2}{|l|}{$\mathrm{Ce}$} & AAA & IM & AHA \\
\hline MAR & OPro & + & - & ++ & +++ & + & ++ & + & - & - & - & - & ++ & - & ++ & +++ & - \\
\hline $\mathrm{AF}$ & OPro & + & + & +++ & ++ & + & + & - & - & - & - & - & + & - & - & ++ & - \\
\hline $\mathrm{AG}$ & OPAll/OPro & + & - & +++ & ++ & - & ++ & - & - & - & - & - & - & - & - & ++ & - \\
\hline MBY & $\mathrm{O} 12$ & ++ & - & + & + & + & + & + & - & - & - & - & - & - & - & - & - \\
\hline $\mathrm{MBC}$ & O14/11/13 & - & - & + & + & - & + & - & + & - & - & + & + & - & + & + & - \\
\hline MFT & 11 & - & - & + & - & - & - & - & - & - & - & - & - & - & + & - & - \\
\hline MBJ & 11 & - & - & + & - & - & - & - & - & - & - & - & - & - & - & - & - \\
\hline $\mathrm{AM}$ & 11 & - & - & + & - & - & - & - & - & - & - & - & - & - & - & - & - \\
\hline \multicolumn{18}{|c|}{ Medial prefrontal } \\
\hline $\mathrm{AH}$ & 25 & + & - & +++ & +++ & +++ & ++ & + & - & + & + & + & - & - & + & + & + \\
\hline AY & 32 & + & - & + & + & + & + & - & - & - & - & + & + & - & - & - & - \\
\hline $\mathrm{AO}$ & M9 & - & - & - & - & - & - & - & - & - & - & - & - & - & - & - & - \\
\hline BA & M10 & - & - & - & - & - & - & - & - & - & - & - & - & - & - & - & - \\
\hline \multicolumn{18}{|c|}{ Lateral prefrontal } \\
\hline $\mathrm{MBH}$ & V46 & - & - & - & - & - & - & - & - & - & - & - & - & - & - & - & - \\
\hline $\mathrm{AA}$ & V46 & - & - & - & - & - & - & - & - & - & - & - & - & - & - & - & - \\
\hline $\mathrm{MFF}$ & V46 & - & - & - & - & - & - & - & - & - & - & - & - & - & - & - & - \\
\hline SF & D10 & - & - & - & - & - & - & - & - & - & - & - & - & - & - & - & - \\
\hline $\mathrm{AB}$ & D46 & - & - & - & - & - & - & - & - & - & - & - & - & - & - & - & - \\
\hline $\mathrm{AD}$ & D8 & - & - & - & - & - & - & - & - & - & - & - & - & - & - & - & - \\
\hline $\mathrm{AC}$ & D8 & - & - & - & - & - & - & - & - & - & - & - & - & - & - & - & - \\
\hline \multicolumn{18}{|c|}{ Temporal cortices } \\
\hline AT & 36 & + & ++ & + & + & ++ & +++ & + & + & ++ & + & + & - & - & + & + & + \\
\hline AV & STG & ++ & + & ++ & + & + & + & - & - & - & - & - & - & - & + & + & - \\
\hline$* \mathrm{BA}$ & $\mathrm{TE}$ & +++ & + & + & - & - & + & - & - & - & - & - & - & - & ++ & + & - \\
\hline \multicolumn{18}{|l|}{ Hypothalamus } \\
\hline $\mathrm{AX}$ & PA & ++ & + & + & + & + & ++ & + & + & + & + & + & +++ & + & + & ++ & + \\
\hline
\end{tabular}

Symbols: - , no label; +, light label (e.g. Fig. 4, BLmc, case MFT); ++, moderate label (e.g. Fig. 3A-C, BLmc, case AG); +++, dense label (e.g. Fig. 3B-C, BLmc, case AF). Abbreviations: A, anterior; D8, dorsal area 8; D10, dorsal area 10; D46, dorsal area 46; d, dorsal; $\mathrm{i}$, intermediate; 1 , lateral; $\mathrm{m}$, medial; M9, medial area 9; mc, magnocellular; pc, parvicellular; P, posterior; v, ventral; V46, ventral area 46. 

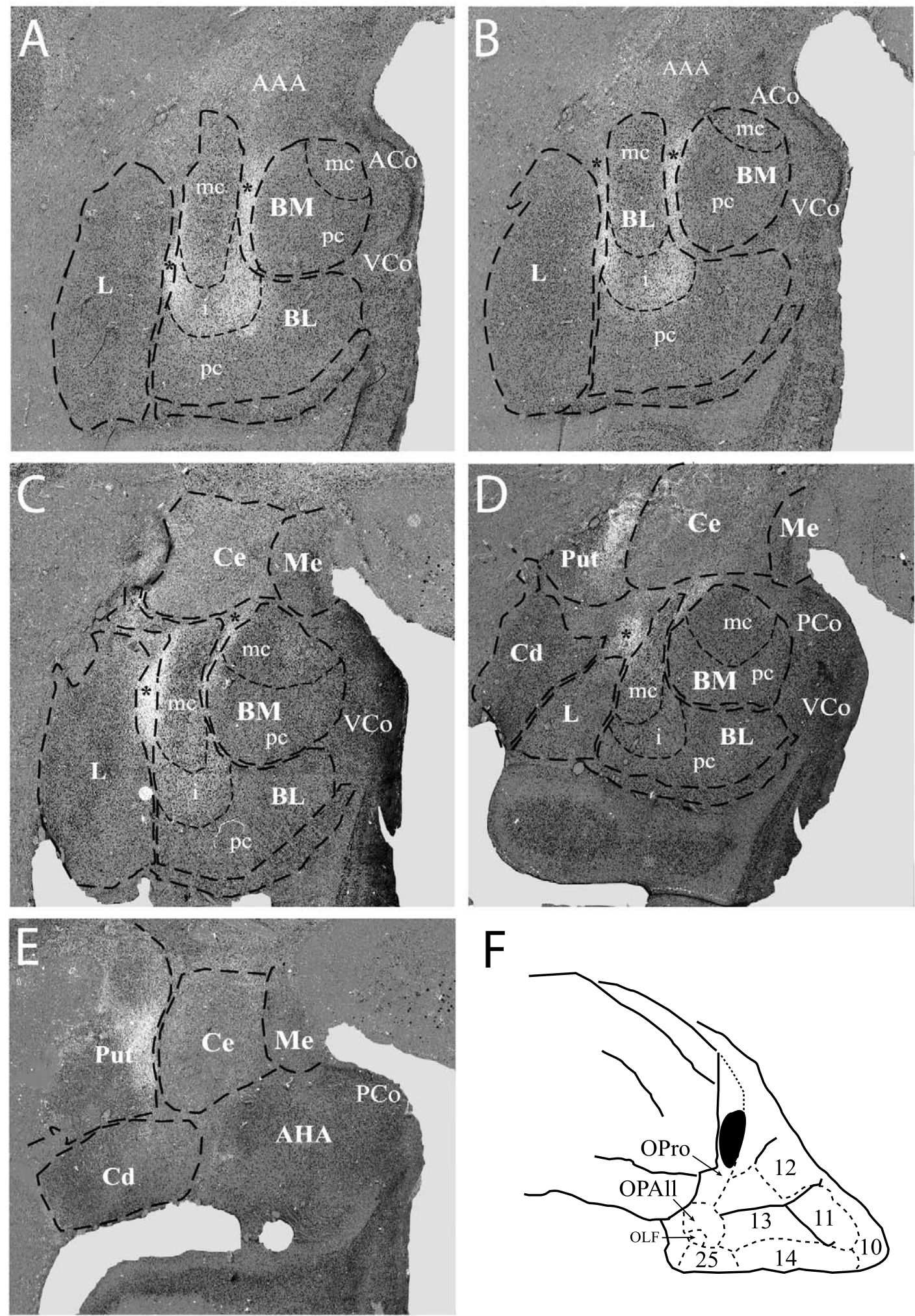

Fig. 2. Caudal orbitofrontal axonal terminations in the amygdala. (A-E) Photomicrographs showing the distribution of axonal terminals (white grain) in rostral (A) to caudal (E) coronal sections through the amygdala after injection of $\left[{ }^{3} \mathrm{H}\right]$ amino acids in orbitofrontal area OPro (F, black area, case MAR). Darkfield/brightfield double exposure to show anterograde label (white grain) as well as the outlines of the nuclei of the amygdala. Black asterisks show label in the IM. Magnification in A-E, $8.5 \times$. 


\section{Case AF}
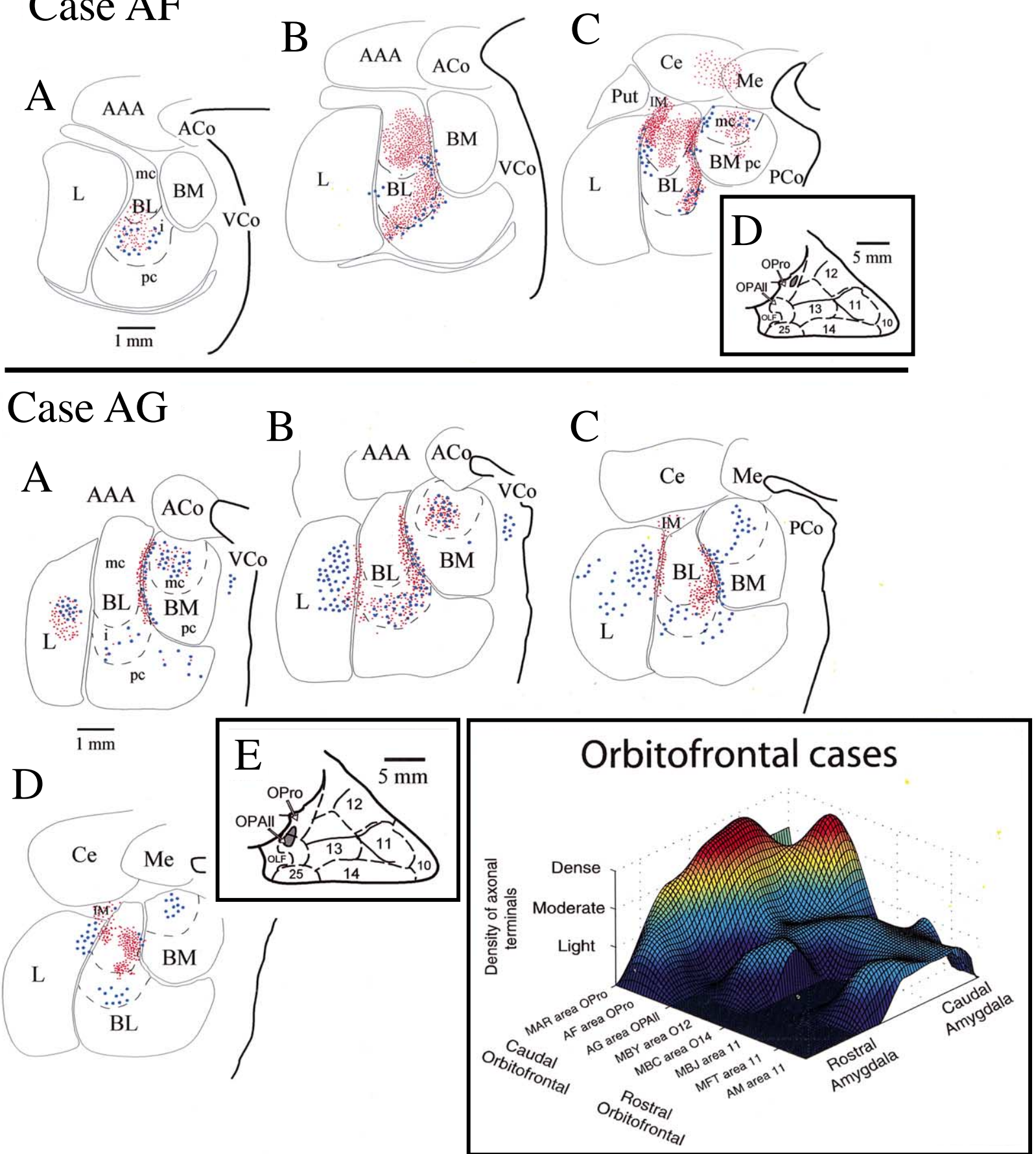

Fig. 3. Pattern of input and output connections linking the amygdala with caudal orbitofrontal areas. Distribution of labeled neurons (large blue dots) and anterograde label (small red dots) in maps of coronal sections in rostral to caudal amygdala mapped after injections of bidirectional tracers in caudal orbitofrontal areas. Top, case AF (A-C): Injection of HRP-WGA was in orbitofrontal area OPro (D, black area). Bottom, case AG (A-D): Injection of HRP-WGA was in orbitofrontal areas OPro and OPAll (E, black area). (Inset) Surface plot showing the density of axonal terminations (vertical axis, from Table 2) along the rostrocaudal extent of the amygdala ( $y$ axis), in all cases with injections in orbitofrontal cortices ( $x$ axis).

O14 was noted in the central region of BL nuclei at the border of its parvicellular and magnocellular sectors (Fig. 4, top, case MBC, A-B; the halo of the injection impinged on adjacent areas 11 and 13, C). Axons from O12 terminated in more nuclei, including all nuclei of the basal complex (L, BL, BM) and the IM (Fig. 4, center, case MBY, A-C; Table 2). In cases with injection in area $11(n=3)$, light anterograde label was mostly limited to the BLmc (Fig. 4, bottom, case MFT; cases MBJ and AM, data not shown; Table 2).

Medial prefrontal termination zones in the amygdala. Axons from medial prefrontal areas 25 and 32 terminated heavily in the caudal half of the amygdala 


\section{Case MBC}

A

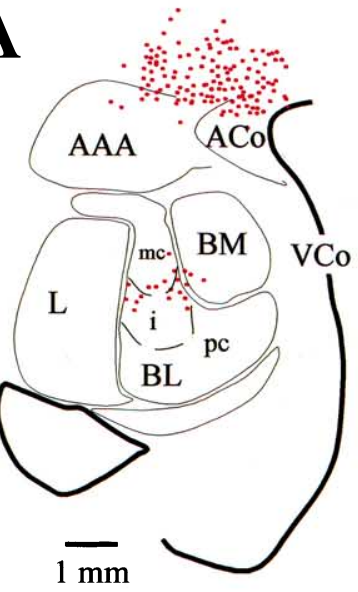

B

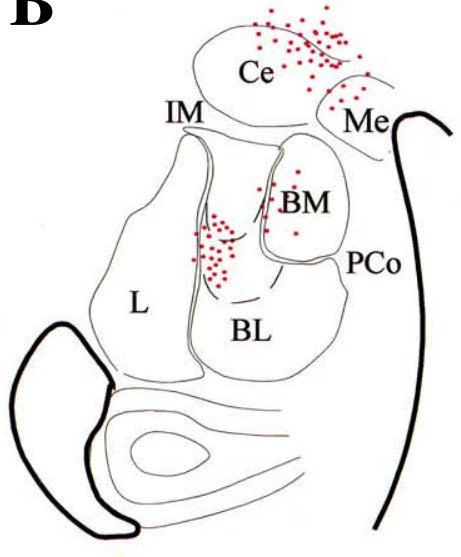

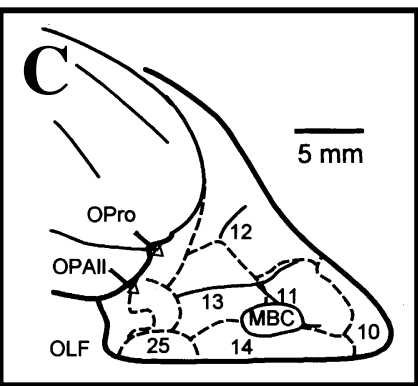

\section{Case MBY}
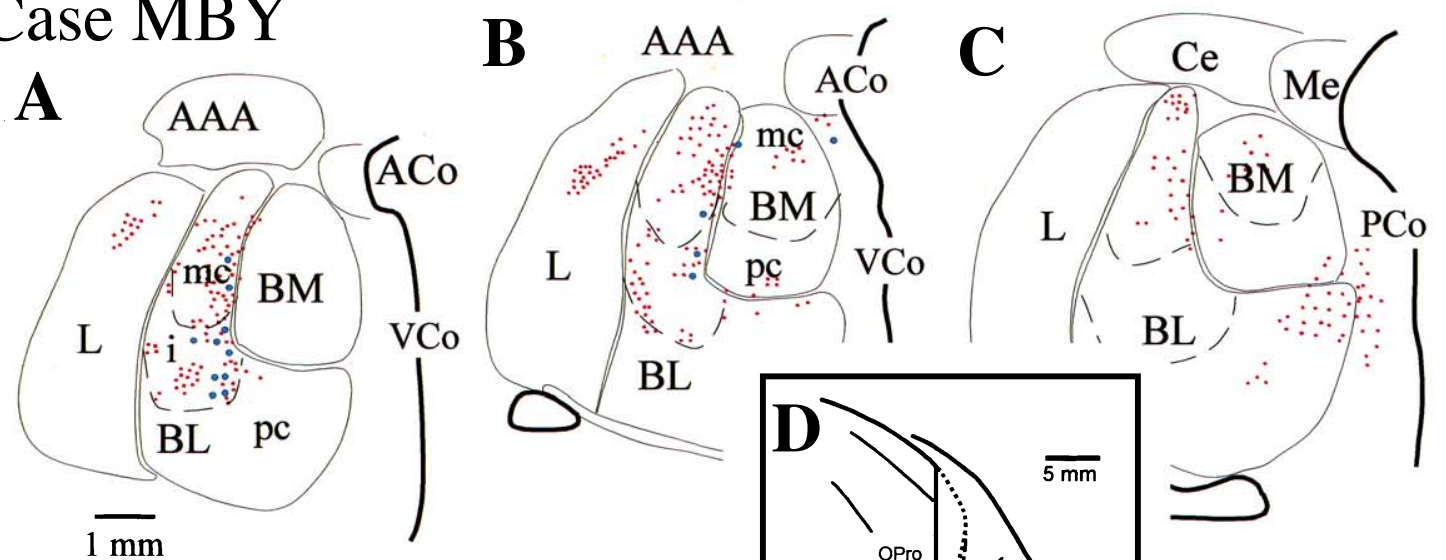

$1 \mathrm{~mm}$

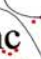

$\mathrm{BM}$

pe $\mathrm{vCo}$

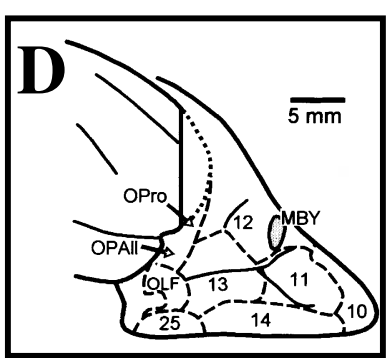

\section{Case MFT}

A
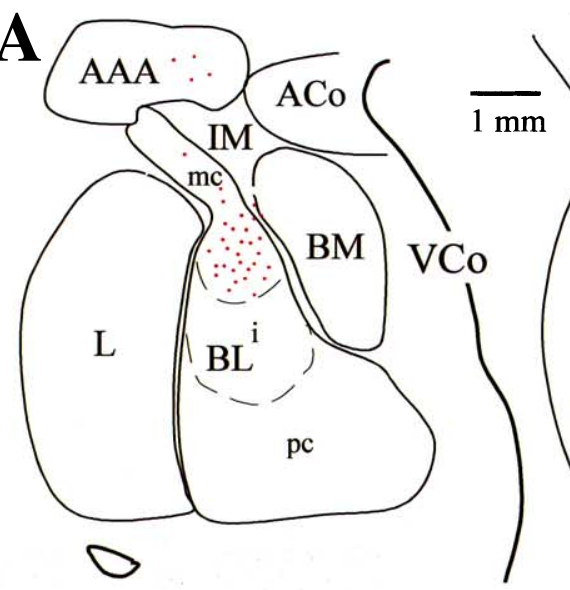

B<smiles>CC1CC2CC3CC1CC(C)(C2)C3</smiles>
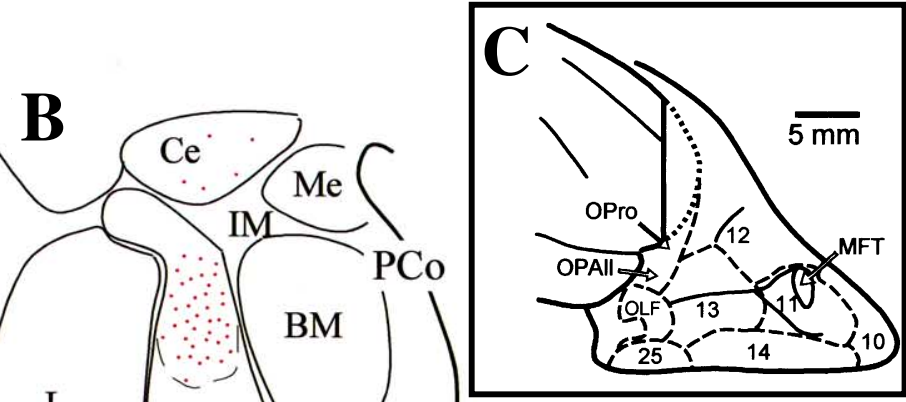

Fig. 4. Connections linking rostral orbitofrontal areas with the amygdala. Diagrams of coronal sections in rostral to caudal amygdala showing, top, case MBC (A, B): Anterograde label (small red dots) after injection of $\left[{ }^{3} \mathrm{H}\right]$ amino acids in area 11 (the halo of the injection impinged on adjacent areas O14 and 13, outlined area, C). Middle, case MBY (A-C): Anterograde label (red dots) and retrogradely labeled neurons (blue dots), after injection of the bidirectional tracer HRP-WGA in O12 (D, gray area). Bottom, case MFT (A, B): Anterograde label after injection of $\left[{ }^{3} \mathrm{H}\right]$ amino acids in area 11 (outlined area, C). 
Case $\mathrm{AH}$

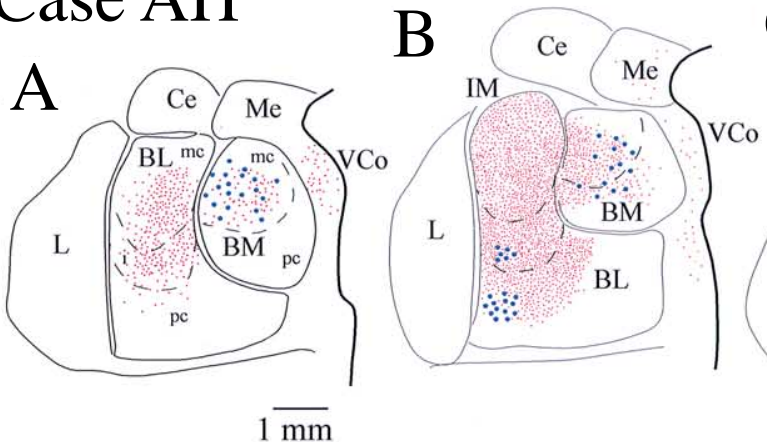

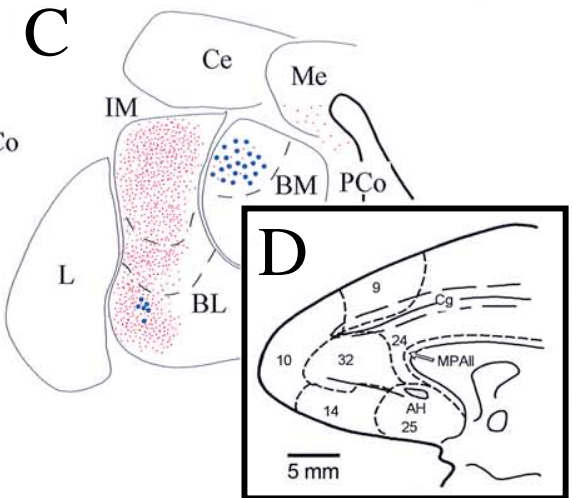

\section{Case AE}

A
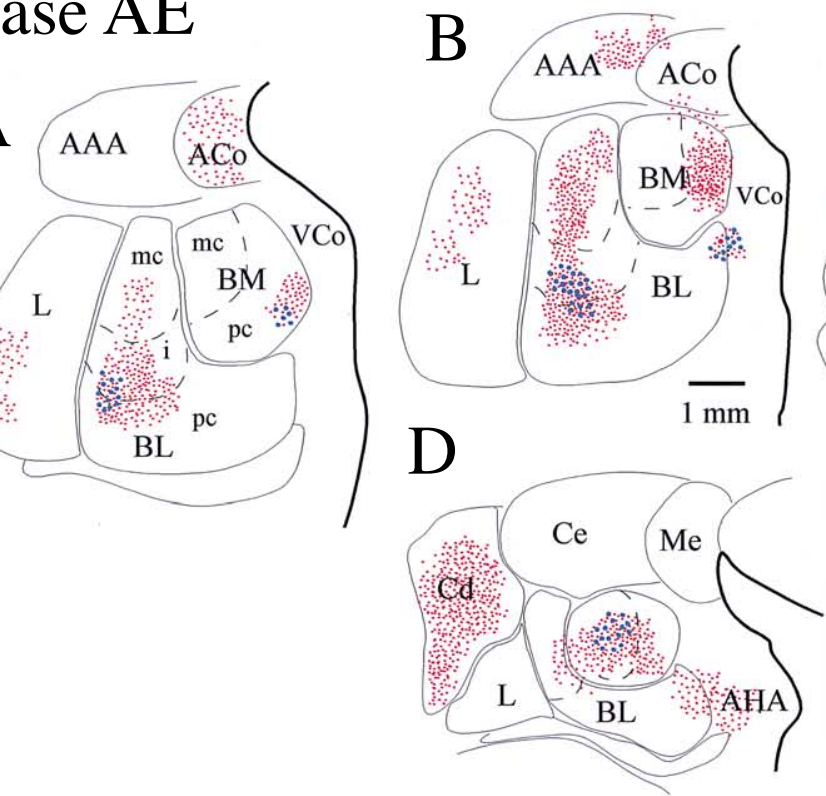

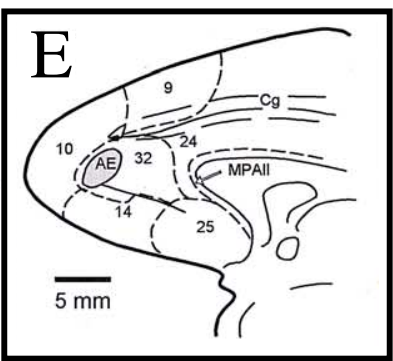

\section{Case AY}

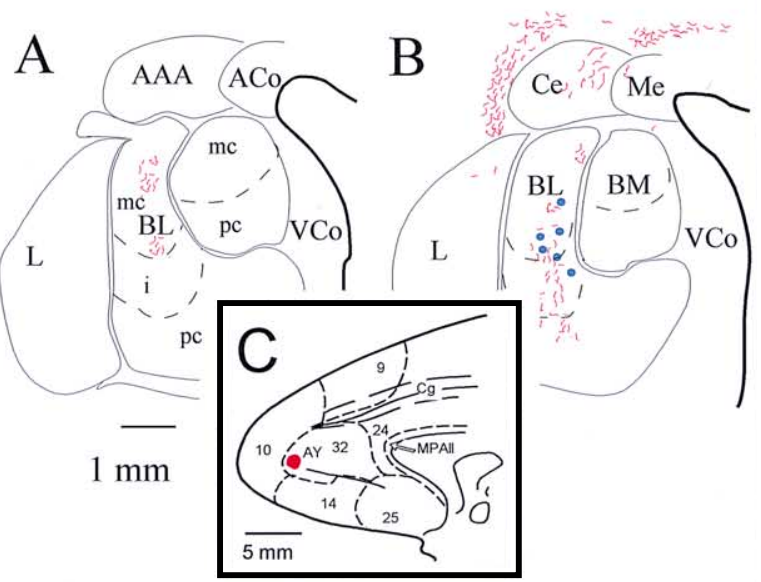

Medial cases

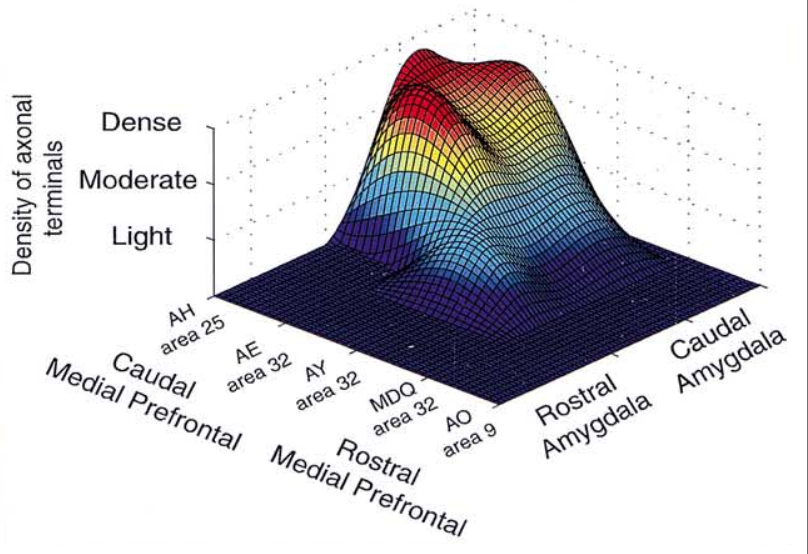

Fig. 5. Pattern of input and output connections linking the amygdala with medial prefrontal areas. Distribution of labeled neurons (large blue dots) and anterograde label (small red dots) in coronal sections in rostral to caudal amygdala mapped after injections of bidirectional tracers in medial prefrontal areas. Top, case AH (A-C): Injection of HRP-WGA was in medial prefrontal area 25 (D, gray area). Middle, case AE (A-D): Injection of HRP-WGA was in medial prefrontal area 32 (E, gray area). Bottom, case AY (A, B): Injection of BDA was in medial prefrontal area 32 (C, red area). (Inset) Surface plot showing the density of axonal terminations (vertical axis, from Table 2) along the rostrocaudal extent of the amygdala $(y$ axis) in all cases with injections in medial prefrontal cortices ( $x$ axis). 

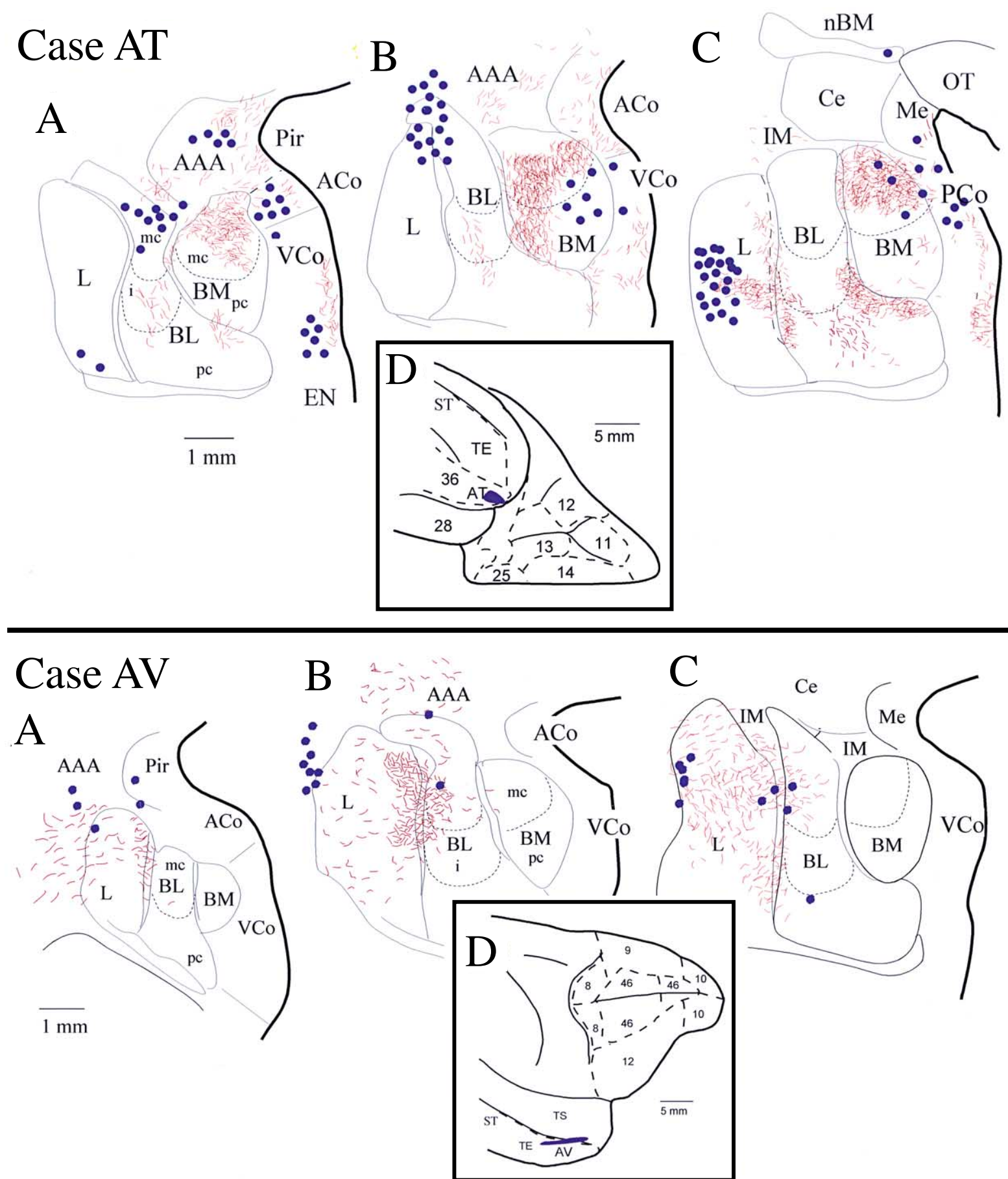

Fig. 6. Pattern of input and output connections linking the amygdala with anterior temporal cortices. Distribution of labeled neurons (blue dots) and anterograde label (brown lines) in a series of coronal sections in rostral to caudal amygdala after injections of BDA in anterior temporal cortical areas. Top, case AT (A-C): Injection of BDA was in temporal area 36 (D, blue area). Bottom, case AV (A-C): Injection of BDA was in temporal areas TE1/TS1 (D, blue area).

(Fig. 5, inset), coinciding in topography with the orbitofrontal zone, but extending into more medial and ventral sites into BLpc and the medial (Me), as well as the cortical (Co) nuclei of the amygdala. Labeled axons from area 25 (case $\mathrm{AH}$ ), in particular, terminated heavily in the BLpc and BLmc, and moderately in BM (Fig. 5, top, case AH, A-C). Light anterograde label was seen medially, including the Me, ventral cortical (VCo) (Fig. 5, top, case $\mathrm{AH}, \mathrm{A}-\mathrm{C}$ ), anterior cortical (ACo) nuclei, AAA, and the amygdalohippocampal area (AHA) (Table 2). A similar pattern of termination was seen after injection in area $32(n=2)$, which also included 
light label of axonal terminals in the medial sector of the Ce nucleus (Fig. 5, center, case AE; bottom, case AY; Table 2).

Lateral prefrontal cortices. In contrast to orbitofrontal and medial prefrontal cortices, injections in lateral prefrontal areas 46, 8, and $10(n=7$, Fig. 1C) did not result in significant anterograde label in the amygdala (Table 2). However retrogradely labeled neurons were present in the BLmc, as reported previously (Barbas and De Olmos, 1990).

Relationship of input to output prefrontal zones in the amygdala. We used bidirectional tracers to determine the relationship of axonal terminals to projection neurons in the amygdala that link it with prefrontal areas. After orbitofrontal injections of HRP-WGA, a dense 'core' of anterogradely labeled axonal terminals extended from the lateral border of BL nucleus to the IM, interposed between the BL and the $\mathrm{L}$ nuclei, clustered at the border of the BLmc and BLpc at the mid-region of the nucleus, and extended dorsally at the medial edge of BLmc, where it borders the accessory basal, or BM nucleus (Fig. 3, cases AF, AG). Labeled neurons formed a 'shell' around the labeled terminals, spanning the borders of the nuclei or subdivisions of the basal complex, along the medial edge of the $\mathrm{L}$, the middle part of $\mathrm{BL}$ and the medial border of BLmc nuclei (Fig. 3, case AF, B-C; case AG, A-D). This evidence suggests at least partial spatial segregation of projection neurons and axonal terminals in the amygdala. The pattern markedly differed after medial prefrontal injections, with clusters of retrogradely labeled neurons lying in a more expansive field of anterogradely labeled terminals, spanning several nuclei, including the BM (accessory basal), BL, VCo, ACo and Me nuclei (Fig. 5).

Connections of anterior temporal cortices with the amygdala: common zones with orbitofrontal and medial prefrontal cortices

Orbitofrontal and medial prefrontal areas are bidirectionally connected with anterior temporal cortices, which, in turn, are heavily connected with the amygdala (e.g. Herzog and Van Hoesen, 1976; Amaral and Price, 1984; Price et al., 1987; Morecraft et al., 1992; Barbas, 1993; Suzuki and Amaral, 1994; Stefanacci et al., 1996; Rempel-Clower and Barbas, 2000; Barbas et al., 1999; Cavada et al., 2000). We compared the relationship of connection zones of anterior temporal cortices in the amygdala with the prefrontal, using two approaches. First, we made a comparison across cases with injection of the primarily anterograde tracer BDA in anterior temporal cortices (cases AT, AV). In one case, the injection was in area 36 (visual and polymodal temporal area; Fig. 6 , case AT, D). Labeled neurons and axonal terminals were noted throughout the rostrocaudal extent of the amygdala, and were most densely distributed in the BM nucleus, particularly in its magnocellular sector (BMmc; Fig. 6, case AT). Columns of labeled axonal terminals were noted along the borders of the BL nucleus
(Fig. 6, case AT, B-C). These findings are consistent with a previous study (Stefanacci et al., 1996). In a second case, the injection of BDA was mostly in the superior temporal gyrus (STG) (auditory association area), but it also extended to the inferior temporal gyrus in visual area TE1 (Fig. 6, bottom, case AV, D). In this case, labeled axonal terminals were seen at the lateral border of BLmc rostrally, and BLpc more caudally (Fig. 6, case AV). The $\mathrm{L}$ nucleus also contained dense anterograde label, particularly in its dorsal part, in agreement with previous findings (Stefanacci and Amaral, 2000). In contrast to the previous case, few, if any, axonal terminals were observed in the BM nucleus.

In a second approach, we used a within-case comparison to directly compare anterior temporal and orbitofrontal zones in the amygdala (Fig. 7). In one animal we simultaneously injected the bidirectional tracer FR in temporal area TE and FB in $\mathrm{O} 12$ (Fig. 7, case BA, D). Axonal terminals from area TE targeted the dorsal borders of the BL nucleus, confirming the findings from the above cases. In the same case, retrogradely labeled neurons projecting to area TE were located in the dorsal aspects of the amygdala, particularly in BLmc and BMmc (Fig. 7, case BA). Axonal terminations from area TE did not overlap with the neurons projecting to O12, reminiscent of the spatial segregation of projection neurons and axonal terminals linking the amygdala with distinct orbitofrontal cortices. Rather, axonal terminals from area TE occupied the same territory as the axonal terminals from caudal orbitofrontal cortex, including the dorsomedial part of the $\mathrm{L}$ nucleus and the central part of the BLmc. Neurons projecting to area TE and O12 constituted largely separate populations, although they were intermingled in the dorsal part of $\mathrm{BL}$ and $\mathrm{BM}$ nuclei (Fig. 7, case BA). In a second case (BC), we placed injections of $\mathrm{FB}$ and $\mathrm{FE}$ in orbitofrontal areas OPro (orbital proisocortex) and 13, and a third injection of FR in temporal auditory association area TS2 of the STG (Fig. 7, case BC, D, E), and found a similar overlap of labeled neurons in the dorsal part of BM that projected to orbitofrontal cortices and the STG. In this case, intermingling of projection neurons was also noted in the L nucleus (Fig. 7, case BC, B-C). Furthermore, though occupying similar sites, neurons projecting to TS2 were a distinct population from those projecting to orbitofrontal areas OPro and 13. There was no evidence of doublelabeled neurons, though the possibility of false negatives cannot be excluded (see also Sripanidkulchai et al., 1984).

As summarized in Fig. 8, the greatest overlap in the connections linking the amygdala with prefrontal cortices and temporal cortices occurred in the posterior half of the amygdala, the region with the highest density of axonal terminals from the prefrontal cortices (Fig. 8C, D). Within this region, the highest degree of overlap was noted in the BMmc, also known as accessory basal, as well as in the intermediate division of the BL nucleus (BLi; Fig. 8C, D). In the BMmc, axonal terminals from visual temporal areas overlapped with projection neurons directed to medial and orbitofrontal cortices, a pattern also seen in a small part of the parvicellular part 


\section{Case BA}

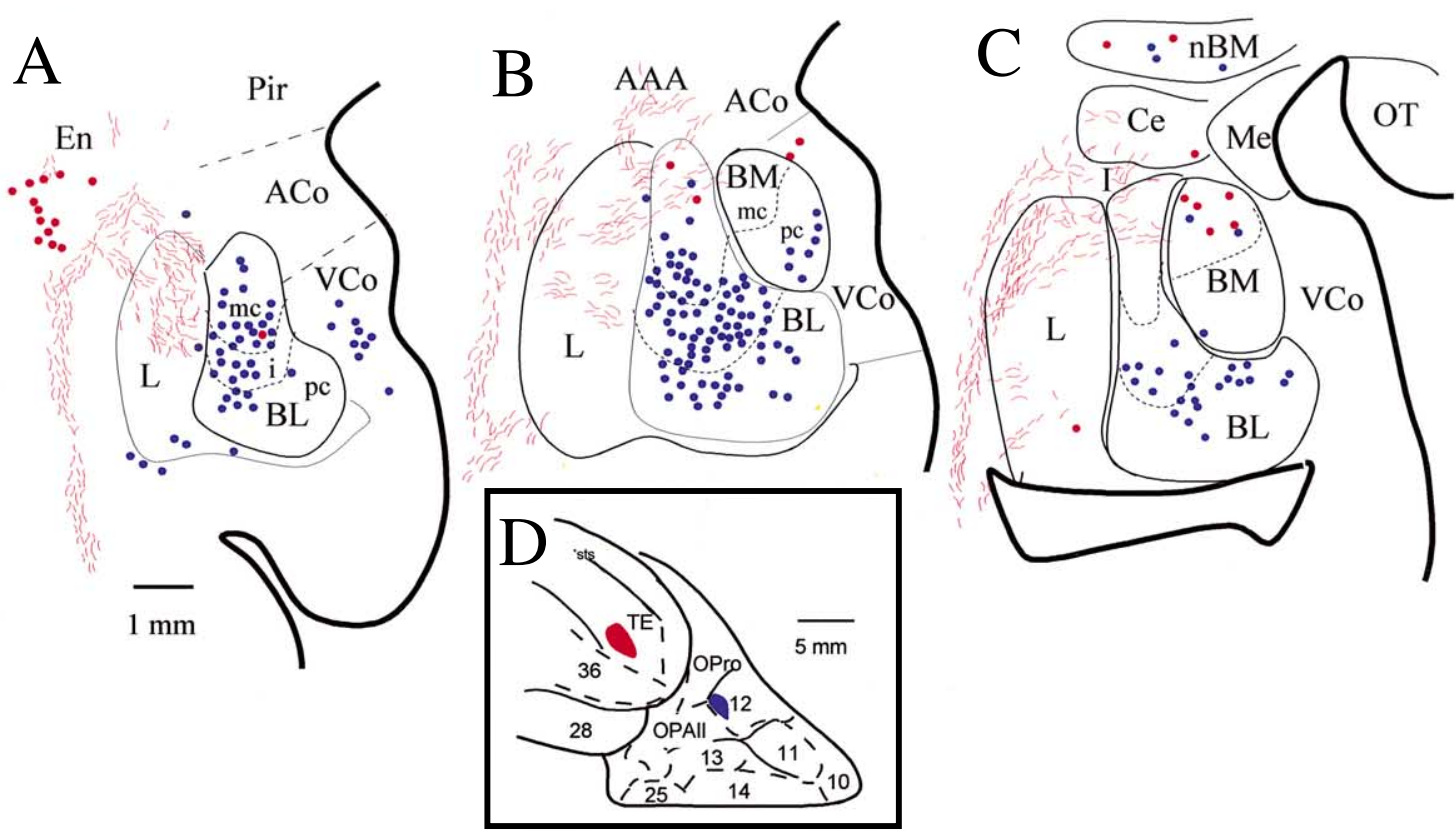

\section{Case BC}

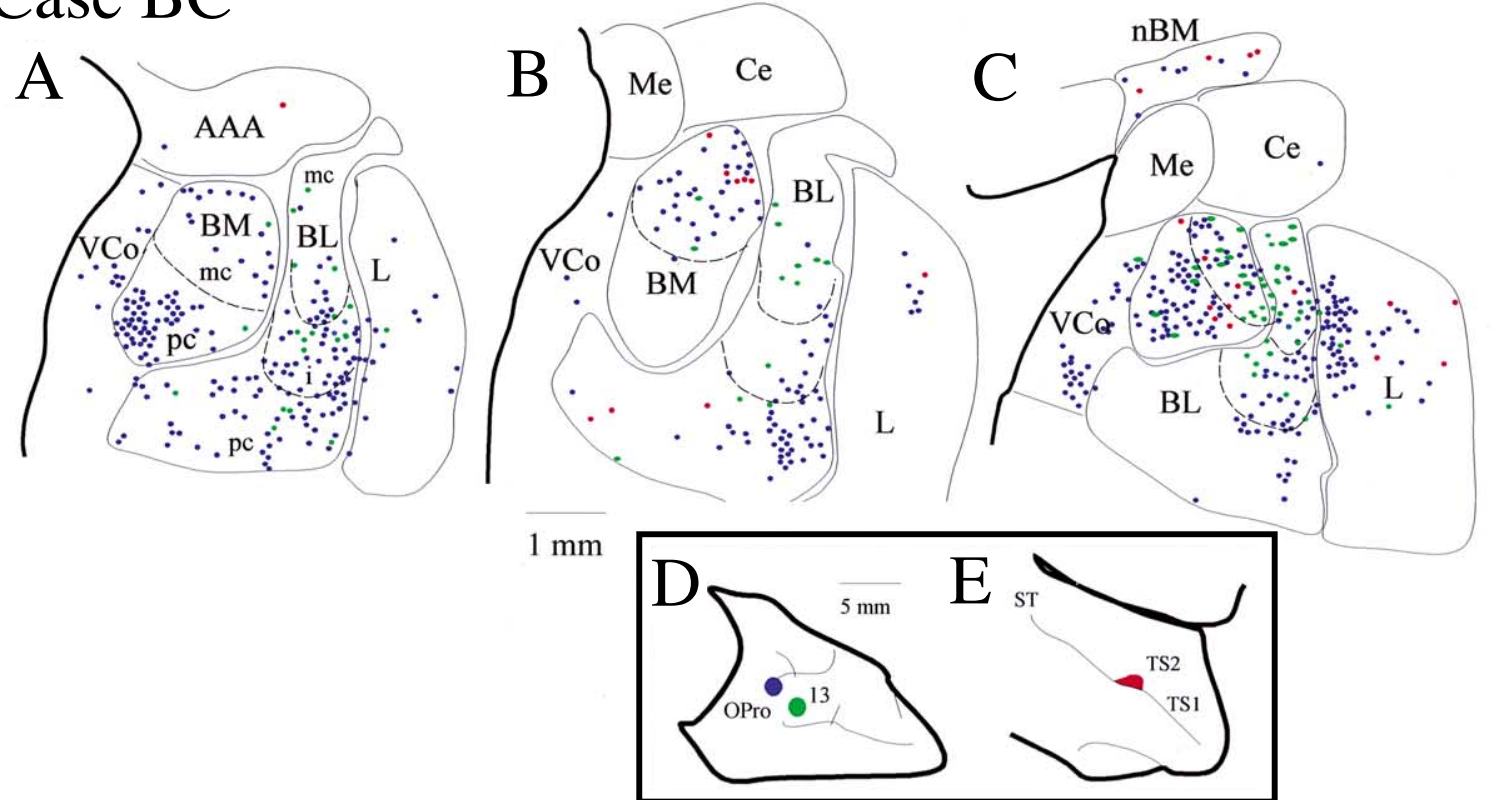

Fig. 7. Comparison of anterior temporal and orbitofrontal connection zones in the amygdala. Top, case BA (A-C): Distribution of labeled neurons projecting to area TE (red dots), and to $\mathrm{O} 12$ (blue dots), and anterograde label from area TE (red lines) in a series of coronal sections in rostral to caudal amygdala after injection of FR in temporal cortical area TE (D, red area) and simultaneous injection of FB in $\mathrm{O} 12$ (D, blue area). Bottom, case BC (A-C): Neurons in the amygdala projecting to prefrontal areas OPro (blue dots) and 13 (green dots) and TS2 (red dots) in coronal sections in rostral to caudal amygdala. (D): Injection of FE was in area 13 (green); FB in OPro, (blue); and (E): FR in TS2 (red).

of $\mathrm{BM}$ nucleus $(\mathrm{BMpc})$ rostrally in the amygdala (Fig. $8 \mathrm{~B})$. The BLi, sandwiched between the BLmc and BLpc, included dense axonal terminations from orbitofrontal and medial prefrontal cortices, projection neurons directed to orbitofrontal cortices, axons from visual areas on its medial and lateral borders (Fig. 8C, D), and to a lesser extent axons from auditory temporal cortices (Fig. 8A, B). In addition, axons from caudal orbitofrontal cortices (but not from medial prefrontal) overlapped with axons from visual and auditory temporal cortices in the IM (Fig. 8B, C). In the posterior half of the amygdala, axons from orbitofrontal cortices were found in the $\mathrm{Ce}$ nucleus by themselves, as were axons and neurons connected with visual temporal cor- 

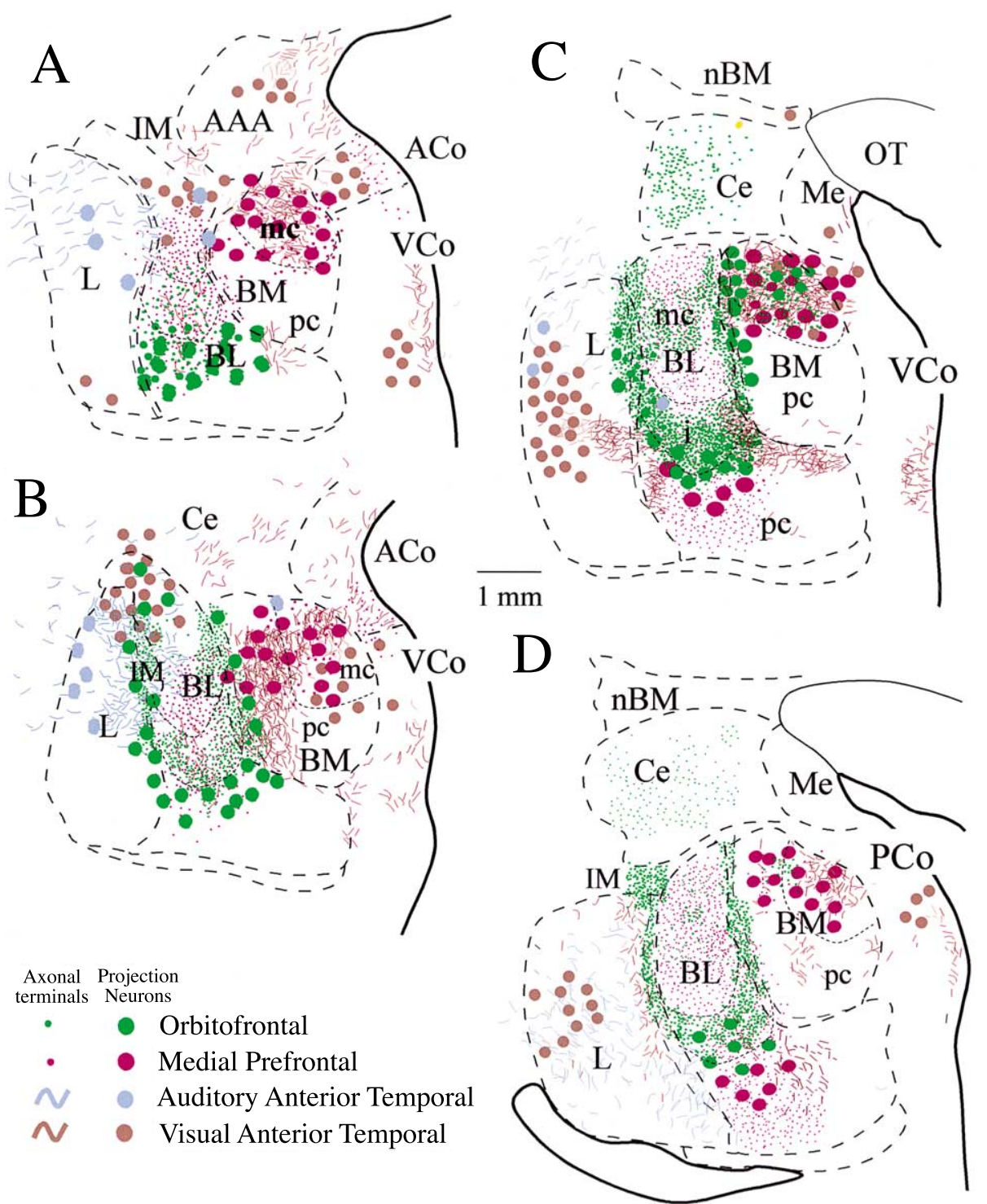

Fig. 8. Zones of overlap in the amygdala linking it with orbitofrontal, medial prefrontal and anterior temporal association cortices. Significant overlap between prefrontal and anterior temporal cortical connections was noted in the caudal half of the amygdala within the $\mathrm{BLi}$, the adjacent part of the BMmc, and the IM, interposed between the borders of BLmc. Approximate rostral to caudal stereotaxic coordinates from a rhesus monkey atlas (Paxinos et al., 2000): A, IA = 19.2 mm, Bregma $=-02.7 \mathrm{~mm} ; \mathrm{B}, \mathrm{IA}=16.95 \mathrm{~mm}$, Bregma $=-04.95 \mathrm{~mm} ; \mathrm{C}, \mathrm{IA}=15.6 \mathrm{~mm}$, Bregma $=-06.3 \mathrm{~mm} ; \mathrm{D}, \mathrm{IA}=14.25 \mathrm{~mm}$, Bregma $=-07.65 \mathrm{~mm}$.

tices in the medial and most sectors of the Co nuclei throughout the rostral to caudal extent of the amygdala (Fig. 8C, D).

Observations on hypothalamic connections with the amygdala: common territory with the medial prefrontal cortices

Orbitofrontal and medial prefrontal cortices are bidirectionally connected with hypothalamic autonomic areas, and all of these structures are connected with the amygdala (for reviews see Price et al., 1987; Barbas et al., 2002). Here we made a few observations on the relationship of zones in the amygdala connected with the hypothalamus and prefrontal termination zones, using an across case approach by placing injections of tracers in hypothalamic nuclei in two animals. After injection of BDA in the PA labeled projection neurons and axonal terminals were distributed in the BLpc, $\mathrm{Ce}, \mathrm{L}$ and $\mathrm{BMmc}$ nuclei (case AX, not shown), and occupied similar sites within the BLpc and BMmc as projection neurons and axonal terminals connected with orbitofrontal and medial prefrontal cortices. In a second animal, after injection of FB in the LA, we noted a large number of retrogradely labeled projection neurons in the $\mathrm{Ce}$ nucleus, confirming findings by previous investigators (for review see Price et al., 1987). Projection neurons were noted also in the BLpc and BMpc (accessory basal), in sites which issue projections to the orbitofrontal cortices, and receive preferential projections from medial prefrontal cortices.

To determine the nature of projection neurons in the 

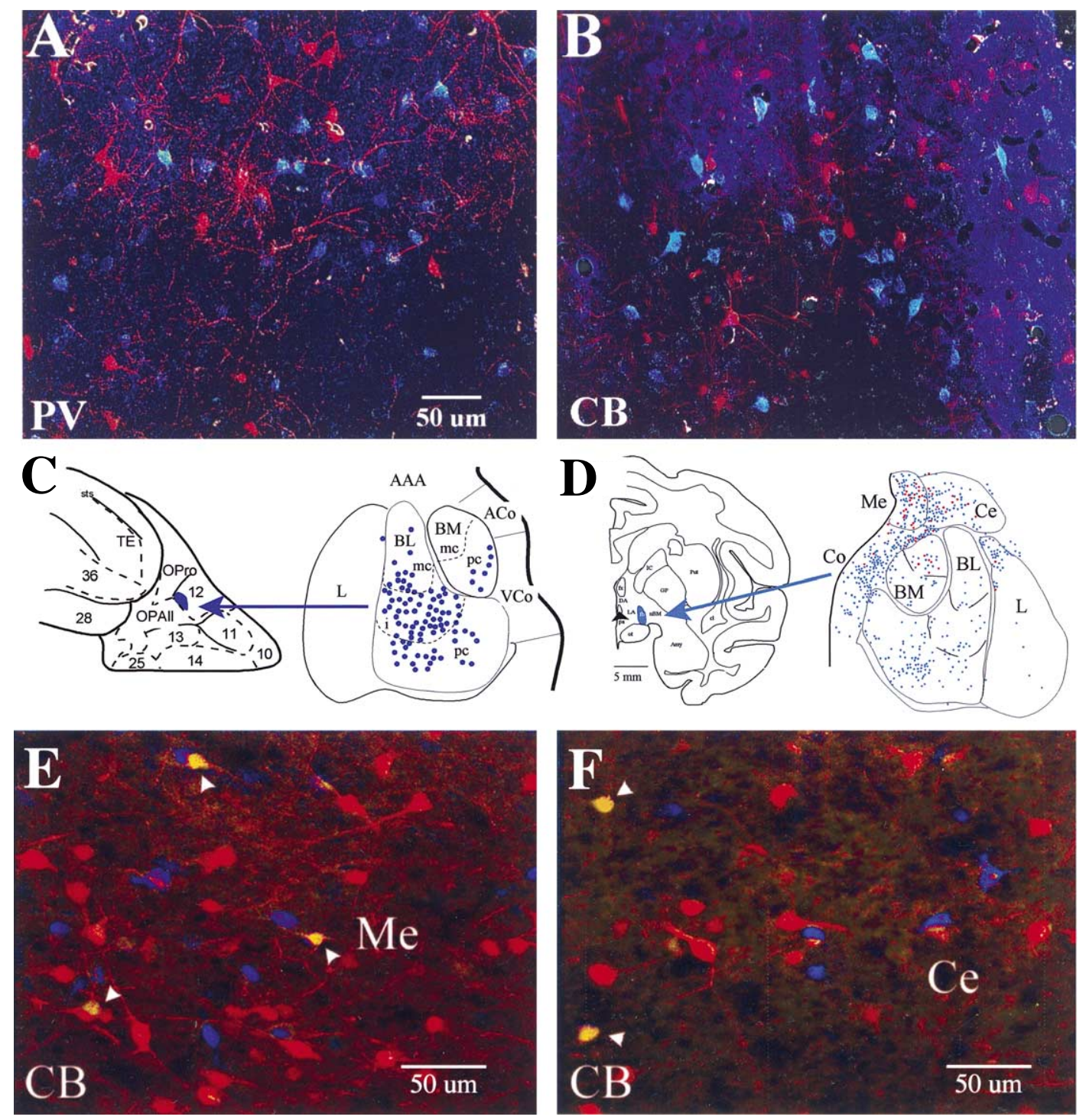

Fig. 9. Relationship of projection neurons to the distribution of neurons positive for two calcium binding proteins. (A) Neurons projecting to prefrontal area $\mathrm{O} 12$ (blue neurons, case BA) were not positive for PV (red neurons); or (B) CB (red neurons). (C) Low power map through the amygdala showing projection neurons directed to area 12 (blue dots). The photomicrographs in (A) and (B) were taken through the BLi of the section in (C). (D) Map of projection neurons (blue, right side) in the amygdala directed to the LA in case AW (blue, left side), and a subpopulation of double-labeled projection neurons that express $\mathrm{CB}$, noted mostly in the Me and Ce nuclei (red dots, right side). (E, F) Photomicrographs of sections through the $\mathrm{Me}$ and $\mathrm{Ce}$ nuclei of the amygdala show a mixed population of projection neurons (blue), single-labeled $\mathrm{CB}$ positive neurons (red) and double-labeled neurons, which projected to the LA and were positive for CB (yellow neurons indicated by white arrow heads). The scale bar in (A) applies to (B).

amygdala and their relationship to local interneurons, we investigated whether they expressed $\mathrm{PV}$ or $\mathrm{CB}$, two markers that largely label distinct classes of inhibitory interneurons in the amygdala and cortex (e.g. Hendry et al., 1989; van Brederode et al., 1990; Lund and Lewis, 1993; Condé et al., 1994; Gabbott and Bacon, 1996; Yan et al., 1996). We conducted this analysis in cases with injections of fluorescent dyes and BDA in anterior temporal, medial prefrontal, orbitofrontal cortices, and the hypothalamus.

Neurons positive for $\mathrm{PV}$ or $\mathrm{CB}$ did not project to the cortex (Fig. 9, case BA, A-C). In contrast, significant numbers of projection neurons directed to the hypothalamus were positive for CB (Fig. 9, case AW, D-F).
Moreover, there were differences in the relative distribution of $\mathrm{CB}$ positive projection neurons that projected to the LA in different nuclei of the amygdala. Of the labeled projection neurons, $20 \%$ were positive for $\mathrm{CB}$ in $\mathrm{Me}$ and BM nuclei (accessory basal), 12\% in the AAA and Ce nuclei, relatively few were noted in the $\mathrm{L}$ and $\mathrm{Co}$ nuclei $(\sim 5 \%)$, and none in the BL nucleus. There was no evidence that projection neurons in the amygdala directed to the hypothalamus were positive for PV. The IM included a large number of projection neurons, some of which were positive for $\mathrm{CB}$ (Fig. 9D). We found no evidence that any of the neurons in the IM that projected to the hypothalamus were positive for PV, which was not expressed in neurons in this region of the amygdala, as 

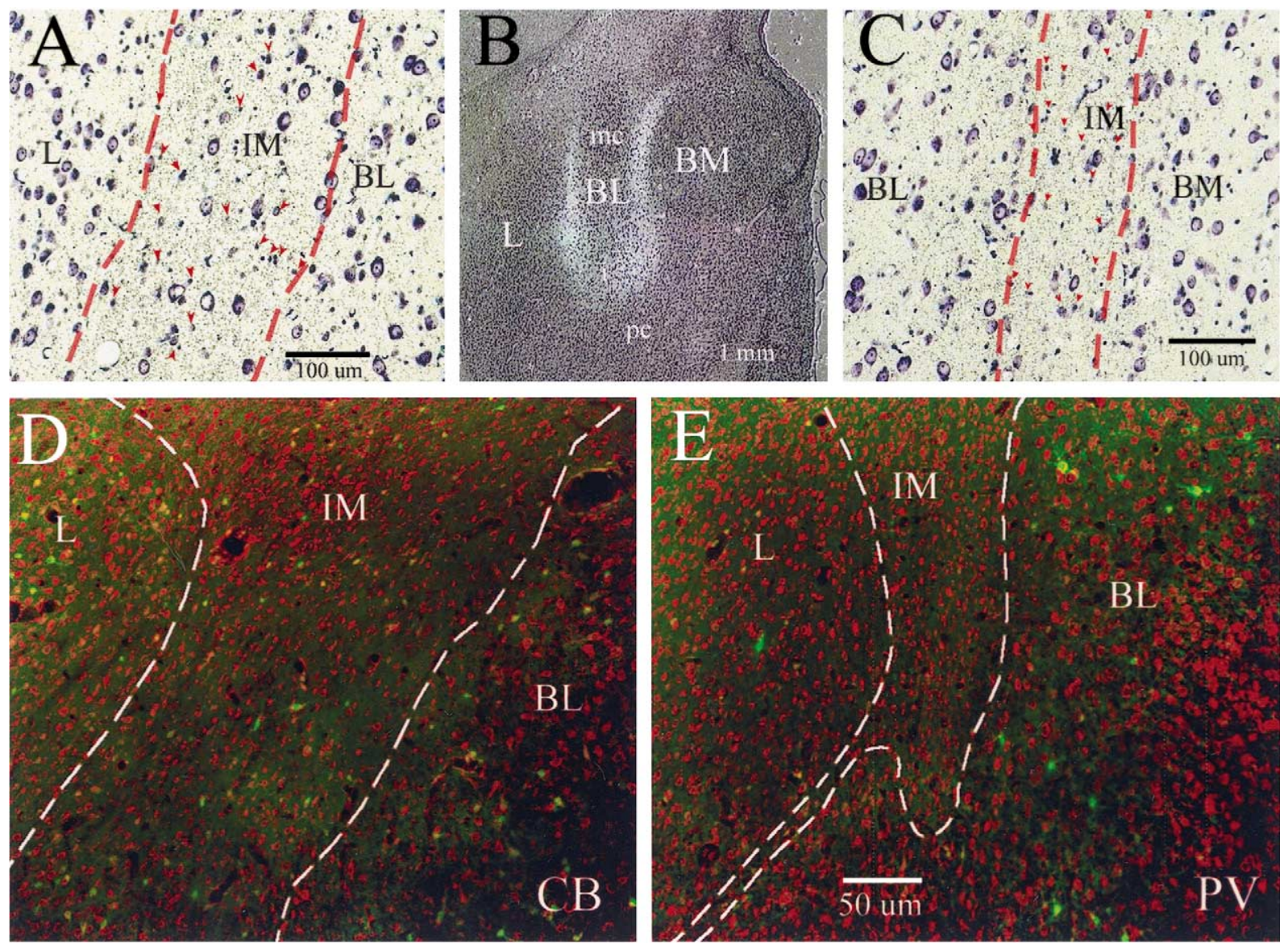

Fig. 10. Focus of axonal terminations from caudal orbitofrontal cortices in the amygdala. (A) High power brightfield photomicrograph (from site in (B)) showing dense terminations of orbitofrontal axons (black grain) in the IM interposed between the $\mathrm{L}$ and $\mathrm{BL}$ nuclei of the amygdala. (B) Low power photomicrograph through the amygdala showing the focus of anterograde label (white label) at the borders of BLmc (case MAR; injection of $\left[{ }^{3} \mathrm{H}\right]$ amino acids was in area OPro; double exposure under brightfield and darkfield illumination). (C) High power brightfield photomicrograph showing dense anterograde label in the IM separating the BL from the BM (accessory basal) nuclei (from site in (B)). (D) CB positive neurons (green) in a region through the IM and the adjacent $\mathrm{L}$ and BL nuclei. (E) PV positive neurons (green) noted in the $\mathrm{L}$ and BL nuclei, but not in the IM of the amygdala. Tissue immunolabeled for PV or CB was counterstained with fluorescent Nissl (red).

(D-E, confocal microscopy)

described previously (Pitkanen and Amaral, 1993) (Fig. $10 \mathrm{E})$.

\section{DISCUSSION}

Partly segregated input-output zones link the amygdala with orbitofrontal cortices

There is strong evidence that the amygdala and the orbitofrontal cortex have an important role in emotions (for reviews see Kling and Steklis, 1976; Aggleton, 1993; Damasio, 1994; Davis, 1997; Roberts and Wallis, 2000; Rolls, 2000; Bechara et al., 2000; Elliott et al., 2000; LeDoux, 2000). Our results indicated that the circuits connecting these structures are bidirectional, suggesting a strong functional tie. Moreover, the connections have spatially distinct input and output zones. Orbitofrontal axons were arranged in a dense U-shaped pattern around the borders of the BLmc, and projection neurons were positioned around the axonal terminations, occupying a continuum at adjacent sites of the basal complex. By comparison, no other prefrontal projection system showed such specific organization in the amygdala. Thus, even though medial prefrontal areas exhibited strong bidirectional connections with the amygdala, their input and output zones were largely intermingled. However, the degree of interaction of input and output zones linking the amygdala with medial as well as with orbitofrontal cortices remains to be determined at the synaptic level. Finally, the connections between the amygdala and lateral prefrontal cortices were modest, largely unidirectional and ascending. Our results confirm and extend previous studies which had primarily focused on the ascending projections (e.g. Porrino et al., 1981; Barbas and De Olmos, 1990; Morecraft et al., 1992).

\section{Orbitofrontal-temporal interactions}

The orbitofrontal cortices and the amygdala process information from every sensory modality (for review and references see Barbas, 1995a). Our findings provided direct evidence that axons from anterior temporal visual and auditory association cortices occupy some of the 
same territories in the amygdala as axons from orbitofrontal cortices, particularly around the borders of the BL nucleus, as summarized in Fig. 8. A significant degree of overlap in the connections of orbitofrontal, medial prefrontal, and temporal visual association areas was noted in the $\mathrm{BLi}$, and in the BM nucleus, including most of its magnocellular division (BMmc) as well as the lateral division of its parvicellular division, where it abuts the central part of the BL nucleus (Fig. 8B). In addition, axons from caudal orbitofrontal cortices overlapped with axons from visual and auditory temporal cortices in the interacalated masses, interposed between the $\mathrm{L}$ and $\mathrm{BL}$ nuclei, and between the BL and BM nuclei. This evidence suggests a closely linked triadic network linking the amygdala, orbitofrontal, and anterior temporal cortices, and implies that sensory input reaches orbitofrontal cortices directly through corticocortical pathways (Rempel-Clower and Barbas, 2000), and indirectly through the amygdala (Barbas, 1995a). This intricate network may form the anatomic substrate for processing the emotional significance of events, in cross-modal associative learning, reward associations, and cognitive tasks that are inextricably linked with emotional associations (Malkova et al., 1997; Hikosaka and Watanabe, 2000; Baxter et al., 2000). In fact, orbitofrontal neurons appear to encode in memory changes in reward contingencies that accompany changes in task demands (Tremblay and Schultz, 2000). Our data suggest that the primary amygdaloid sites of such influence are within the basal complex and the surrounding intercalated neurons.

Autonomic related pathways in the prefrontal-amygdaloid axis: emotional expression

The amygdala is further involved in emotional expression and accompanying autonomic, endocrine, and vegetative responses mediated by projections of $\mathrm{Ce}, \mathrm{Co}$ and Me nuclei to autonomic structures (e.g. Price and Amaral, 1981; Price et al., 1987; De Olmos, 1990; Gray, 1993). The latter innervate peripheral organs, including the lungs, the heart and the gut, whose activity is markedly changed in emotional situations. Emotional expression encompasses a variety of bodily changes ranging from conscious motor responses to vegetative, autonomic and endocrine responses (e.g. Davis, 1997). Our findings suggest that although axons from medial and orbitofrontal cortices overlapped in the amygdala, those from medial prefrontal areas clearly occupied a wider territory, extending into the BLpc, the Me, and Co nuclei, which project to the hypothalamus.

The type of neurons from BLpc projecting to the hypothalamus is not known, although in our material they were not labeled by either PV or CB, known markers of inhibitory interneurons in the amygdala (e.g. Kemppainen and Pitkanen, 2000; McDonald and Mascagni, 2001). Since we noted that significant numbers of projection neurons directed to the hypothalamus expressed $\mathrm{CB}$ in the $\mathrm{Me}$ and the $\mathrm{Ce}$ nuclei in the same tissue, it is unlikely that their absence in BL nucleus can be attributed to technical factors. This evidence suggests that these projection neurons may be excitatory, but does not preclude the possibility that yet another class of inhibitory interneurons may project from $\mathrm{BL}$ nucleus to the hypothalamus, such as those known to be positive for neuropeptides (e.g. McDonald et al., 1995). However, there is no evidence that $\mathrm{BL}$ nucleus neurons projecting outside the amygdala are inhibitory in primates, though they have been reported in rats (e.g. Morys et al., 1999). If confirmed to be excitatory through physiologic study, then medial prefrontal projections to the amygdala may amplify the output of the BLpc to the hypothalamus, a pathway that may be recruited in concert with a robust direct projection from medial prefrontal cortices to hypothalamic autonomic centers (Leichnetz and Astruc, 1976; Room et al., 1985; Rempel-Clower and Barbas, 1998; Ongur et al., 1998; Freedman et al., 2000). In addition, we noted that medial prefrontal axons terminated in the $\mathrm{Co}$ and $\mathrm{Me}$ nuclei, engaged in the expressive capacity of the amygdala in gustatory, olfactory, and reproductive functions (for review see De Olmos, 1990).

Orbitofrontal cortices appear to influence the output of the amygdala through pathways that differ anatomically and perhaps in mechanism of action. Unlike axons from medial prefrontal areas, orbitofrontal axons terminated heavily in the IM of the amygdala, interposed between the L and BL nuclei (Fig. 10A-C). The neurons in this dense and narrow nucleus are small in size, are largely GABAergic, and project to the Ce nucleus of the amygdala, the basal forebrain, and the brainstem, at least in rats and cats (Moga and Gray, 1985; Paré and Smith, 1993, 1994). In our material we saw CB (but not PV) positive neurons in the IM, consistent with findings from another study (Pitkanen and Amaral, 1993). If most of these neurons are, in fact, inhibitory, then caudal orbitofrontal cortices could have tight control of the internal processing of the amygdala. The $\mathrm{Ce}$ nucleus issues inhibitory projections to the hypothalamus and brainstem (Jongen-Relo and Amaral, 1998; Saha et al., 2000), suggested also by our findings that CB positive neurons projected to the hypothalamus. Upon activation through massive orbitofrontal projections, intercalated neurons can suppress activity in the Ce nucleus and release its inhibitory output to the hypothalamus and brainstem. Suppression of the Ce nucleus may allow time for integration of sensory signals so that an adequate judgement can be made regarding the recruitment of autonomic sectors of the amygdala. Thus, the orbitofrontal pathway likely has a permissive effect on the hypothalamus, whose activity could be further augmented by direct excitatory projections from the same orbitofrontal and medial prefrontal cortices to hypothalamic autonomic centers (Rempel-Clower and Barbas, 1998). It is envisioned that dual pathways with disinhibitory or excitatory effects on the hypothalamus may be recruited when the synergistic activation of the amygdala and the orbitofrontal cortex signals an emotionally charged circumstance.

Caudal orbitofrontal areas also issued a direct, though considerably weaker, projection to the Ce nucleus, than to the IM. Activation of this pathway may result in fast return to autonomic homeostasis through inhibition of 
the hypothalamus and brainstem, when emotional arousal subsides. Our data thus suggest a dual role of orbitofrontal axons in the amygdala, positioned either to increase or decrease the drive to the Ce nucleus, depending on the nature of integrated sensory input processed in the amygdala and orbitofrontal cortices.

Our findings also showed convergence of axonal terminals from the hypothalamus with efferents from temporal and prefrontal cortices in the dorsal part of BL nucleus, which projects to the frontal eye fields, premotor and motor cortices (for review see De Olmos, 1990). This evidence suggests that the dorsal part of BL nucleus interfaces with structures associated with conscious motor commands, which is distinct from the pathway through the $\mathrm{Ce}$ nucleus affecting autonomic responses.

\section{CONCLUSION}

The unique role of the amygdala and the orbitofrontal cortex in emotions is not understood. The amygdala increases its activity when viewing masked fearful faces, when there is no awareness of the event (Whalen et al., 1998). Moreover, a subcortical loop connecting the amygdala with the thalamus can support fear conditioning (Romanski and LeDoux, 1992). Since the experience of emotions likely involves the cortex (Kennard, 1945), the robust bidirectional pathways linking the amygdala with orbitofrontal cortices may underlie this process. We provided direct evidence that the latter pathway shares territory with temporal sensory association cortices in distinct zones of the basal nuclei of the amygdala, and may be concerned with the cognitive aspect of emotions and the formation of associations between prefrontal, temporal and hypothalamic inputs related to emotional memory. In addition, axonal terminals from orbitofrontal cortices targeted the IM. The latter have been shown to gate information exchange between the $\mathrm{BL}$ and $\mathrm{Ce}$ nuclei in the amygdala, at least in cats (Royer et al., 1999), which likely underlies cognitive processes prior to emotional expression. This pathway appears to be distinct from those involved in the expression of emotions (e.g. Kaada, 1972). The two systems likely interact in behavior in linking emotionally relevant sensory input with emotional expression, in processes entailing learning and memory of emotionally significant associations, interfacing with frontal premotor systems for action, and with autonomic centers for emotional expression. The anatomic evidence suggests that while the orbitofrontal connections with the amygdala may underlie cognitive evaluation of the environment and emotional memory, the medial prefrontal connections are directly, widely, and preferentially linked with the expressive and emotional motor system of the amygdala.

Acknowledgements-We thank Ms. Karen Trait for help with immunocytochemical assays, Dr. Ron Killiany for assistance with MR imaging, and Dr. Deepak Pandya for the autoradiographic cases. This work was supported by grants from NIH (NIMH and NINDS).

\section{REFERENCES}

Aggleton, J.P., 1993. The contribution of the amygdala to normal and abnormal emotional states. Trends Neurosci. 16, $328-333$.

Aggleton, J.P., Burton, M.J., Passingham, R.E., 1980. Cortical and subcortical afferents to the amygdala of the rhesus monkey (Macaca mulatta). Brain Res. 190, 347-368.

Alheid, G.F., Heimer, L., 1996. Theories of basal forebrain organization and the 'emotional motor system'. Prog. Brain Res. $107,461-484$.

Amaral, D.G., Price, J.L., 1984. Amygdalo-cortical projections in the monkey (Macaca fascicularis). J. Comp. Neurol. 230 , $465-496$.

Barbas, H., 1993. Organization of cortical afferent input to orbitofrontal areas in the rhesus monkey. Neuroscience $56,841-864$.

Barbas, H., 1995a. Anatomic basis of cognitive-emotional interactions in the primate prefrontal cortex. Neurosci. Biobehav. Rev. 19 , 499-510.

Barbas, H., 1995b. Pattern in the cortical distribution of prefrontally directed neurons with divergent axons in the rhesus monkey. Cereb. Cortex 5, $158-165$.

Barbas, H., 2000. Connections underlying the synthesis of cognition, memory, and emotion in primate prefrontal cortices. Brain Res. Bull. 52, 319-330.

Barbas, H., Blatt, G.J., 1995. Topographically specific hippocampal projections target functionally distinct prefrontal areas in the rhesus monkey. Hippocampus 5, 511-533.

Barbas, H., De Olmos, J., 1990. Projections from the amygdala to basoventral and mediodorsal prefrontal regions in the rhesus monkey. J. Comp. Neurol. 301, 1-23.

Barbas, H., Ghashghaei, H., Dombrowski, S.M., Rempel-Clower, N.L., 1999. Medial prefrontal cortices are unified by common connections with superior temporal cortices and distinguished by input from memory-related areas in the rhesus monkey. J. Comp. Neurol. 410 , $343-367$.

Barbas, H., Ghashghaei, H., Rempel-Clower, N., Xiao, D., 2002. Anatomic basis of functional specialization in prefrontal cortices in primates. In: Grafman, J. (Ed.), Handbook of Neuropsychology. Elsevier Science B.V., Amsterdam, pp. 1-27.

Barbas, H., Henion, T.H., Dermon, C.R., 1991. Diverse thalamic projections to the prefrontal cortex in the rhesus monkey. J. Comp. Neurol. 313, 65-94.

Barbas, H., Pandya, D.N., 1989. Architecture and intrinsic connections of the prefrontal cortex in the rhesus monkey. J. Comp. Neurol. 286, 353375.

Barbas, H., Rempel-Clower, N., 1997. Cortical structure predicts the pattern of corticocortical connections. Cereb. Cortex 7, 635-646.

Baxter, M.G., Parker, A., Lindner, C.C., Izquierdo, A.D., Murray, E.A., 2000. Control of response selection by reinforcer value requires interaction of amygdala and orbital prefrontal cortex. J. Neurosci. 20, 4311-4319.

Bechara, A., Damasio, H., Damasio, A.R., 2000. Emotion, decision making and the orbitofrontal cortex. Cereb. Cortex $10,295-307$.

Bechara, A., Tranel, D., Damasio, H., Damasio, A.R., 1996. Failure to respond autonomically to anticipated future outcomes following damage to prefrontal cortex. Cereb. Cortex 6, 215-225.

Carmichael, S.T., Price, J.L., 1995a. Limbic connections of the orbital and medial prefrontal cortex in macaque monkeys. J. Comp. Neurol. 363, 615-641. 
Carmichael, S.T., Price, J.L., 1995b. Sensory and premotor connections of the orbital and medial prefrontal cortex of macaque monkeys. J. Comp. Neurol. 363, 642-664.

Cavada, C., Company, T., Tejedor, J., Cruz-Rizzolo, R.J., Reinoso-Suarez, F., 2000. The anatomical connections of the macaque monkey orbitofrontal cortex. A review. Cereb. Cortex 10, 220-242.

Chiba, T., Kayahara, T., Nakano, K., 2001. Efferent projections of infralimbic and prelimbic areas of the medial prefrontal cortex in the Japanese monkey, Macaca fuscata. Brain Res. 888, 83-101.

Condé, F., Lund, J.S., Jacobowitz, D.M., Baimbridge, K.G., Lewis, D.A., 1994. Local circuit neurons immunoreactive for calretinin, calbindin D-28k or parvalbumin in monkey prefrontal cortex: distribution and morphology. J. Comp. Neurol. 341, 95-116.

Cowan, W.M., Gottlieb, D.I., Hendrickson, A.E., Price, J.L., Woolsey, T.A., 1972. The autoradiographic demonstration of axonal connections in the central nervous system. Brain Res. 37, 21-51.

Damasio, A.R., 1994. Descarte's Error: Emotion, Reason, and the Human Brain. G.P. Putnam's Sons, New York.

Damasio, A.R., Tranel, D., Damasio, H., 1990. Individuals with sociopathic behavior caused by frontal damage fail to respond autonomically to social stimuli. Behav. Brain Res. 41, 81-94.

Davis, M., 1997. Neurobiology of fear responses: the role of the amygdala. J. Neuropsychiatry Clin. Neurosci. 9, 382-402.

De Olmos, J., 1990. Amygdaloid nuclear gray complex. In: Paxinos, G. (Ed.), The Human Nervous System. Academic Press, San Diego, pp. 583710.

Dermon, C.R., Barbas, H., 1994. Contralateral thalamic projections predominantly reach transitional cortices in the rhesus monkey. J. Comp. Neurol. 344, 508-531.

Elliott, R., Dolan, R.J., Frith, C.D., 2000. Dissociable functions in the medial and lateral orbitofrontal cortex: evidence from human neuroimaging studies. Cereb. Cortex 10, 308-317.

Freedman, L.J., Insel, T.R., Smith, Y., 2000. Subcortical projections of area 25 (subgenual cortex) of the macaque monkey. J. Comp. Neurol. 421, $172-188$.

Gabbott, P.L., Bacon, S.J., 1996. Local circuit neurons in the medial prefrontal cortex (areas 24a, b, c, 25 and 32) in the monkey: I. Cell morphology and morphometrics. J. Comp. Neurol. 364, 567-608.

Galaburda, A.M., Pandya, D.N., 1983. The intrinsic architectonic and connectional organization of the superior temporal region of the rhesus monkey. J. Comp. Neurol. 221, 169-184.

Gallagher, M., Holland, P.C., 1994. The amygdala complex: multiple roles in associative learning and attention. Proc. Natl. Acad. Sci. USA 91, $11771-11776$.

Gallyas, F., 1979. Silver staining of myelin by means of physical development. Neurol. Res. 1, 203-209.

Geneser-Jensen, F.A., Blackstad, T.W., 1971. Distribution of acetyl cholinesterase in the hippocampal region of the guinea pig. Z. Zellforsch. Mikrosk. Anat. 114, 460-481.

Ghashghaei, H.T., Barbas, H., 2001. Neural interaction between the basal forebrain and functionally distinct prefrontal cortices in the rhesus monkey. Neuroscience 103, 593-614.

Gray, T.S., 1993. Amygdaloid CRF pathways. Role in autonomic, neuroendocrine, and behavioral responses to stress. Ann. N. Y. Acad. Sci. 697, 53-60.

Hendry, S.H.C., Jones, E.G., Emson, P.C., Lawson, D.E.M., Heizmann, C.W., Streit, P., 1989. Two classes of cortical GABA neurons defined by differential calcium binding protein immunoreactivities. Exp. Brain Res. 76, 467-472.

Herzog, A.G., Van Hoesen, G.W., 1976. Temporal neocortical afferent connections to the amygdala in the rhesus monkey. Brain Res. 115, 57-69.

Hikosaka, K., Watanabe, M., 2000. Delay activity of orbital and lateral prefrontal neurons of the monkey varying with different rewards. Cereb. Cortex 10, 263-271.

Jacobson, S., Trojanowski, J.Q., 1975. Amygdaloid projections to prefrontal granular cortex in rhesus monkey demonstrated with horseradish peroxidase. Brain Res. 100, 132-139.

Jongen-Relo, A.L., Amaral, D.G., 1998. Evidence for a GABAergic projection from the central nucleus of the amygdala to the brainstem of the macaque monkey: a combined retrograde tracing and in situ hybridization study. Eur. J. Neurosci. 10, 2924-2933.

Kaada, B.R., 1972. Stimulation and regional ablation of the amygdaloid complex with reference to functional representation. In: Eleftheriou, B.E. (Ed.), The Neurobiology of the Amygdala. Plenum Press, New York, pp. 145-204.

Kemppainen, S., Pitkanen, A., 2000. Distribution of parvalbumin, calretinin, and calbindin-D28k immunoreactivity in the rat amygdaloid complex and colocalization with gamma-aminobutyric acid. J. Comp. Neurol. 426, 441-467.

Kennard, M.A., 1945. Focal autonomic representation in the cortex and its relation to sham rage. J. Neuropathol. Exp. Neurol. 4, $295-304$.

Kling, A., Steklis, H.D., 1976. A neural substrate for affiliative behavior in nonhuman primates. Brain Behav. Evol. 13, $216-238$.

LeDoux, J.E., 2000. Emotion circuits in the brain. Annu. Rev. Neurosci. 23, 155-184.

Leichnetz, G.R., Astruc, J., 1976. The efferent projections of the medial prefrontal cortex in the squirrel monkey (Saimiri sciureus). Brain Res. 109, 455-472.

Loewy, A.D., 1991. Forebrain nuclei involved in autonomic control. Prog. Brain Res. 87, 253-268.

Lund, J.S., Lewis, D.A., 1993. Local circuit neurons of developing and mature macaque prefrontal cortex: Golgi and immunocytochemical characteristics. J. Comp. Neurol. 328, 282-312.

Malkova, L., Gaffan, D., Murray, E.A., 1997. Excitotoxic lesions of the amygdala fail to produce impairment in visual learning for auditory secondary reinforcement but interfere with reinforcer devaluation effects in rhesus monkeys. J. Neurosci. 17, 6011-6020.

McDonald, A.J., 1998. Cortical pathways to the mammalian amygdala. Prog. Neurobiol. 55, 257-332.

McDonald, A.J., Mascagni, F., 2001. Colocalization of calcium-binding proteins and GABA in neurons of the rat basolateral amygdala. Neuroscience $105,681-693$.

McDonald, A.J., Mascagni, F., Augustine, J.R., 1995. Neuropeptide Y and somatostatin-like immunoreactivity in neurons of the monkey amygdala. Neuroscience 66, 959-982.

Mesulam, M.-M., Hegarty, E., Barbas, H., Carson, K.A., Gower, E.C., Knapp, A.G., Moss, M.B., Mufson, E.J., 1980. Additional factors influencing sensitivity in the tetramethyl benzidine method for horseradish peroxidase neurohistochemistry. J. Histochem. Cytochem. 28, $1255-1259$.

Moga, M.M., Gray, T.S., 1985. Peptidergic efferents from the intercalated nuclei of the amygdala to the parabrachial nucleus in the rat. Neurosci. Lett. $61,13-18$

Morecraft, R.J., Geula, C., Mesulam, M.-M., 1992. Cytoarchitecture and neural afferents of orbitofrontal cortex in the brain of the monkey. J. Comp. Neurol. 323, 341-358.

Morys, J., Berdel, B., Kowianski, P., Majak, K., Tarnawski, M., Wisniewski, H.M., 1999. Relationship of calcium-binding protein containing neurons and projection neurons in the rat basolateral amygdala. Neurosci. Lett. 259, 91-94.

Nauta, W.J.H., 1961. Fibre degeneration following lesions of the amygdaloid complex in the monkey. J. Anat. 95, 515-531.

Ongur, D., An, X., Price, J.L., 1998. Prefrontal cortical projections to the hypothalamus in macaque monkeys. J. Comp. Neurol. 401, 480-505. 
Pandya, D.N., Van Hoesen, G.W., Domesick, V.B., 1973. A cingulo-amygdaloid projection in the rhesus monkey. Brain Res. 61, $369-373$.

Paré, D., Smith, Y., 1993. The intercalated cell masses project to the central and medial nuclei of the amygdala in cats. Neuroscience 57 , $1077-$ 1090 .

Paré, D., Smith, Y., 1994. GABAergic projection from the intercalated cell masses of the amygdala to the basal forebrain in cats. J. Comp. Neurol. 344, 33-49.

Paxinos, G., Huang, X.-F., Toga, A.W., 2000. The Rhesus Monkey Brain: In Stereotaxic Coordinates. Academic Press, San Diego.

Pitkanen, A., Amaral, D.G., 1993. Distribution of calbindin-D28k immunoreactivity in the monkey temporal lobe: the amygdaloid complex. J. Comp. Neurol. 331, 199-224.

Porrino, L.J., Crane, A.M., Goldman-Rakic, P.S., 1981. Direct and indirect pathways from the amygdala to the frontal lobe in rhesus monkeys. J. Comp. Neurol. 198, 121-136.

Price, J.L., Amaral, D.G., 1981. An autoradiographic study of the projections of the central nucleus of the monkey amygdala. J. Neurosci. 1, $1242-1259$.

Price, J.L., Russchen, F.T., Amaral, D.G., 1987. The limbic region. II. The amygdaloid complex. In: Björklund, A., Hökfelt, T., Swanson, L.W. (Eds.), Handbook of Chemical Neuroanatomy Vol. 5, Integrated Systems of the CNS, Part I. Elsevier, Amsterdam, pp. $279-381$.

Rempel-Clower, N.L., Barbas, H., 1998. Topographic organization of connections between the hypothalamus and prefrontal cortex in the rhesus monkey. J. Comp. Neurol. 398, 393-419.

Rempel-Clower, N.L., Barbas, H., 2000. The laminar pattern of connections between prefrontal and anterior temporal cortices in the Rhesus monkey is related to cortical structure and function. Cereb. Cortex 10, 851-865.

Roberts, A.C., Wallis, J.D., 2000. Inhibitory control and affective processing in the prefrontal cortex: neuropsychological studies in the common marmoset. Cereb. Cortex 10, 252-262.

Rolls, E.T., 2000. The orbitofrontal cortex and reward. Cereb. Cortex 10, 284-294.

Romanski, L.M., LeDoux, J.E., 1992. Equipotentiality of thalamo-amygdala and thalamo-cortico-amygdala circuits in auditory fear conditioning. J. Neurosci. 12, 4501-4509.

Room, P., Russchen, F.T., Groenewegen, H.J., Lohman, A.H., 1985. Efferent connections of the prelimbic (area 32) and the infralimbic (area 25) cortices: an anterograde tracing study in the cat. J. Comp. Neurol. 242, 40-55.

Royer, S., Martina, M., Paré, D., 1999. An inhibitory interface gates impulse traffic between the input and output stations of the amygdala. J. Neurosci. 19, 10575-10583.

Saha, S., Batten, T.F., Henderson, Z., 2000. A GABAergic projection from the central nucleus of the amygdala to the nucleus of the solitary tract: a combined anterograde tracing and electron microscopic immunohistochemical study. Neuroscience 99, $613-626$.

Saper, C.B., 1990. Hypothalamus. In: Paxinos, G. (Ed.), The Human Nervous System. Academic Press, Boston, pp. $389-413$.

Schoenbaum, G., Chiba, A.A., Gallagher, M., 2000. Changes in functional connectivity in orbitofrontal cortex and basolateral amygdala during learning and reversal training. J. Neurosci. 20, 5179-5189.

Sripanidkulchai, K., Sripanidkulchai, B., Wyss, J.M., 1984. The cortical projection of the basolateral amygdaloid nucleus in the rat: a retrograde fluorescent dye study. J. Comp. Neurol. 229, 419-431.

Stefanacci, L., Amaral, D.G., 2000. Topographic organization of cortical inputs to the lateral nucleus of the macaque monkey amygdala: a retrograde tracing study. J. Comp. Neurol. 421, 52-79.

Stefanacci, L., Suzuki, W.A., Amaral, D.G., 1996. Organization of connections between the amygdaloid complex and the perirhinal and parahippocampal cortices in macaque monkeys. J. Comp. Neurol. 375, 552-582.

Suzuki, W.A., Amaral, D.G., 1994. Perirhinal and parahippocampal cortices of the macaque monkey: Cortical afferents. J. Comp. Neurol. 350, 497-533.

Tremblay, E., Schultz, W., 2000. Reward-related neuronal activity during go-nogo task performance in primate orbitofrontal cortex. J. Neurophysiol. $83,1864-1876$.

van Brederode, J.F., Mulligan, K.A., Hendrickson, A.E., 1990. Calcium-binding proteins as markers for subpopulations of GABAergic neurons in monkey striate cortex. J. Comp. Neurol. 298, 1-22.

Van Hoesen, G.W., 1981. The differential distribution, diversity and sprouting of cortical projections to the amygdala of the rhesus monkey. In: Ben-Ari, Y. (Ed.), The Amygdaloid Complex. Elsevier/North Holland Biomedical Press, Amsterdam, pp. 77-90.

Whalen, P.J., Rauch, S.L., Etcoff, N.L., McInerney, S.C., Lee, M.B., Jenike, M.A., 1998. Masked presentations of emotional facial expressions modulate amygdala activity without explicit knowledge. J. Neurosci. 18, 411-418.

Yan, X.X., Jen, L.S., Garey, L.J., 1996. NADPH-diaphorase-positive neurons in primate cerebral cortex colocalize with GABA and calciumbinding proteins. Cereb. Cortex 6, 524-529. 